ARTICLE

\title{
Toxoplasma LIPIN is essential in channeling host lipid fluxes through membrane biogenesis and lipid storage
}

Sheena Dass ${ }^{1,4}$, Serena Shunmugam (10 1,4, Laurence Berry ${ }^{2}$, Christophe-Sebastien Arnold ${ }^{1}$, Nicholas J. Katris ${ }^{1}$, Samuel Duley ${ }^{1}$, Fabien Pierrel [D $^{3}$, Marie-France Cesbron-Delauw ${ }^{1}$, Yoshiki Yamaryo-Botté ${ }^{1,5 凶}$ \& Cyrille Y. Botté (i) ${ }^{1,5}$ 凶

Apicomplexa are obligate intracellular parasites responsible for major human diseases. Their intracellular survival relies on intense lipid synthesis, which fuels membrane biogenesis. Parasite lipids are generated as an essential combination of fatty acids scavenged from the host and de novo synthesized within the parasite apicoplast. The molecular and metabolic mechanisms allowing regulation and channeling of these fatty acid fluxes for intracellular parasite survival are currently unknown. Here, we identify an essential phosphatidic acid phosphatase in Toxoplasma gondii, TgLIPIN, as the central metabolic nexus responsible for controlled lipid synthesis sustaining parasite development. Lipidomics reveal that TgLIPIN controls the synthesis of diacylglycerol and levels of phosphatidic acid that regulates the fine balance of lipids between storage and membrane biogenesis. Using fluxomic approaches, we uncover the first parasite host-scavenged lipidome and show that TgLIPIN prevents parasite death by 'lipotoxicity' through effective channeling of host-scavenged fatty acids to storage triacylglycerols and membrane phospholipids.

\footnotetext{
${ }^{1}$ Apicolipid Team, Institute for Advanced Biosciences, CNRS UMR5309, Université Grenoble Alpes, INSERM U1209, Grenoble, France. ${ }^{2}$ Laboratory of Pathogen Host Interactions, UMR 5235, Université de Montpellier, Montpellier, France. ${ }^{3}$ Université Grenoble Alpes, CNRS, Grenoble INP, TIMC-IMAG, Grenoble, France. ${ }^{4}$ These authors contributed equally: Sheena Dass, Serena Shunmugam. ${ }^{5}$ These authors jointly supervised this work: Yoshiki Yamaryo-Botté, Cyrille Y. Botté. ${ }^{凶}$ email: yoshiki.botte-yamaryo@univ-grenoble-alpes.fr; cyrille.botte@univ-grenoble-alpes.fr
} 
A picomplexa includes several pathogenic protists that are responsible for major chronic and infectious diseases with a massive social and economic burden. Toxoplasma gondii and Plasmodium falciparum parasites are responsible for toxoplasmosis and malaria, respectively. These obligate intracellular parasites have an enormous demand for lipids in maintaining their survival within their human host cells. The utilization of fatty cids (FA) to synthesize complex lipids is an essential determinant for successful host adaptation by these parasites. Apicomplexa meet their high need for lipids through de novo synthesis via type II fatty acid synthesis (FASII) within the apicoplast and via copious salvage directly from the host and extracellular environment ${ }^{1-6}$. Recent data suggest that the tight regulation of FA flux between host, parasite, and its metabolic organelles is particularly vital for intracellular development of these apicomplexa pathogens ${ }^{4,7}$. Metabolic adaptation of the parasite toward host nutritional environment is also important for parasite propagation ${ }^{4}$. For instance, in low nutrient environments, the parasite is able to form giant multivesicular bodies (gMVBs) in the host, which somehow facilitates the parasite's ability to scavenge lipids in suboptimal nutritional conditions ${ }^{4}$. However, the molecular and metabolic pathways controlling the lipid flux toward parasite membrane biogenesis and storage remain largely unknown.

Phosphatidic acid (PA), the simplest glycerophospholipid, contributes to the regulation of FA flux in apicomplexa, as it is the key intermediate balancing the biosynthesis of both glycerophospholipids and triacylglycerols (TAGs) toward membrane biogenesis and lipid storage in eukaryotes ${ }^{8}$. PA has pleiotropic roles within the parasite: (i) as a signal transducer modulating parasite invasion, motility, and egress, produced by the action of diacylglycerol kinases (DGK1 and DGK2) ${ }^{9-12}$, (ii) as a regulator of lysoPA (LPA)/PA levels for modulating membrane curvature for cytokinesis and endocytosis, formed by acyltransferase ATS2 4 , and (iii) as the central precursor for bulk phospholipid synthesis and membrane biogenesis, made de novo from FA and a glycerol3 -phosphate backbone by the sequential acylation catalyzed by acyltransferases, in the apicoplast/ER pathways formed by ATS1/ GPAT and ATS2/AGPAT ${ }^{4,13}$. Further, PA can be catabolized to a key lipid class, diacylglycerol (DAG) by phosphatidic acid phosphatases (PAP), which play vital metabolic functions in eukaryotes ${ }^{14}$. Together, PA and DAG are central lipid precursors from which most lipid classes can be generated de novo. This includes all phospholipids made from PA and DAG via the CDPDAG from $P A$ and the Kennedy pathway from DAG, respectively ${ }^{15,16}$. DAG is also the precursor for the synthesis of the major storage lipids, TAG.

Since PA is pivotal for parasite lipid metabolism, the enzymes involved in PA metabolism are critical for understanding parasite pathogenesis. One such critical enzyme class is PAP, as it allows to fine-tune the balance between PA and DAG. Apicomplexa parasites possess three putative PAPs, none of which have been characterized to date.

Here, we determined that Toxoplasma gondii LIPIN; TgLIPIN is a PAP that localizes to the cytosolic-ER interface and is essential for the intracellular survival of tachyzoites. Its inducible disruption quickly leads to aberrant membrane anomalies, mostly at the IMC and the endomembrane system, causing division defects and parasite death. Lipidomics of the mutant reveals a time-dependent accumulation of PA and free FA (FFA), concomitant with the reduction of DAG, lipid droplets, and storage, TAG. Both lipidomic and cellular membrane phenotypes are aggravated in a high host nutrient environment. The mutant dies from a lipotoxic accumulation of FFA and major membrane phospholipids, specifically PA. Further, we conducted novel fluxomic experiments using $\mathrm{U}_{-}{ }^{13} \mathrm{C}$ glucose-labeled host cells to monitor the host FA scavenging capacity of the parasite, providing the first host-scavenged lipidome of Toxoplasma. This approach showed that TgLIPIN channels host FA to maintain an appropriate PA/DAG synthesis ratio. TgLIPIN acts as a metabolic checkpoint, tightly regulating membrane biogenesis versus lipid storage, which is essential for proper intracellular growth division and survival.

\section{Results}

Toxoplasma gondii genome encodes a single lipin, TgLIPIN, which has functional phosphatidate phosphatase activity. Bioinformatics analysis revealed that $T$. gondii parasites possess a single lipin homolog, much larger than other phosphatidate phosphatases encoded by the genome of T. gondii, and which we named TgLIPIN (TGGT1_230690). TgLIPIN possesses the two typical and highly conserved domains of eukaryotic lipins, the amino-terminal N-LIP domain, and the carboxy-terminal C-LIP domain harboring its functional PA phosphatase catalytic motif DXDXT/V (HAD-like domain, Fig. 1a) ${ }^{17}$. Phylogenetic analysis confirms that the enzyme is highly conserved as a single lipin within phylum Apicomplexa, cladding specifically within a coccidian subgroup (Supplementary Fig. 1a).

To confirm the predicted phosphatidic acid phosphatase activity of TgLIPIN (Fig. 1b), we performed heterologous complementation using a Saccharomyces cerevisiae triple mutant, $\Delta d p p 1 \Delta l p p 1 \Delta p a h 1$, that is deficient in PAP activity with a temperature-sensitive phenotype (low growth at $\left.37^{\circ} \mathrm{C}\right)^{18,19}$. We attempted to generate the full $T g L I P I N$ sequence and the corresponding recombinant protein but were not able to do so, due to limitations posed by the large size of the protein. Instead, we successfully generated TgLIPIN C-LIP domain, containing the putative catalytic (HAD-like) domain of the protein, and expressed it in the yeast mutant. We thus conducted lipidomic analyses on the yeast mutant, complemented or not with TgLIPIN C-LIP domain. The yeast mutant displayed a severe and significant accumulation of PA and a significant reduction of DAG compared to the wild-type yeast strain, corresponding to the loss of PA phosphatase activity (Fig. 1c). Complementation of the yeast mutant with the C-LIP domain of TgLIPIN fully restored the levels of PA and DAG to those of the wild type, thus confirming its predicted PAP activity (Fig. 1b, c).

To determine its cellular localization, $T g L I P I N$ was endogenously tagged with $3 \times \mathrm{HA}$ at its C-terminal end ${ }^{20}$. Immunofluorescence assay (IFA) and colocalization with known organelle markers revealed a broad cytosolic and perinuclear localization (Supplementary Fig. 1b). Interestingly TgLIPIN lacks an apparent nuclear localization signal (NLS), possessed by most eukaryotic $L I P I N s^{8,21}$. To further resolve its localization, we conducted IFAs of TgLIPIN-HA with a known Toxoplasma ER marker, DER-1$\mathrm{GFP}^{22}$ an episomally expressing plasmid, which confirmed proximity to the endomembrane system with partial ER colocalization (Fig. 1d). TgLIPIN was not further detected outside the parasite or in the host cell (Supplementary Fig. 1b).

TgLIPIN disruption induces rapid membrane malformation, division, and replication defects leading to parasite death, all aggravated under a high host nutrient environment. To understand the importance of TgLIPIN in parasite growth, we generated an N-terminal HA-tagged inducible knockdown parasite line based on the Tet-off system, TgLIPIN-ikD 23,24 (Supplementary Fig. 2a, b). Downregulation of TgLIPIN-ikD showed no detectable protein by western blot after $48 \mathrm{~h}$ of anhydrotetracycline (ATc) treatment (Fig. 2a). Cytoplasmic localization of the protein in the same manner as C-terminally tagged strains and its downregulation were both confirmed by IFA (Fig. 2b). This result 
a

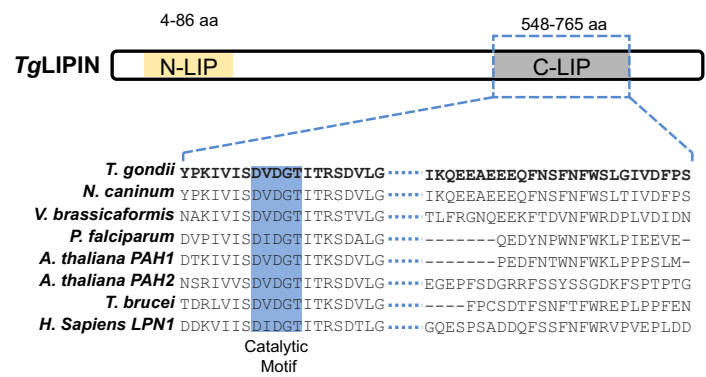

C

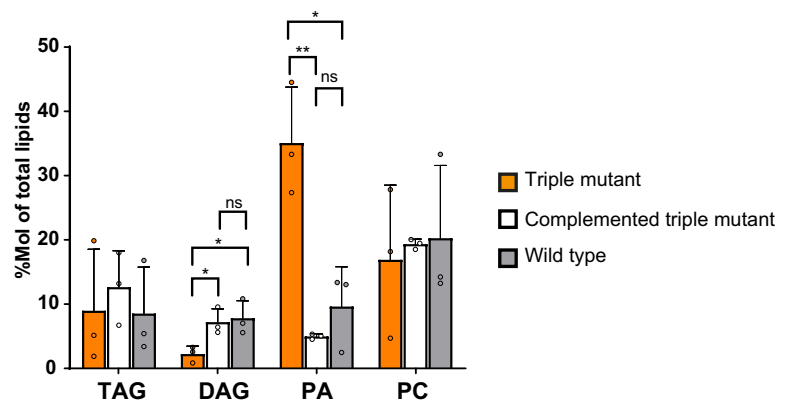

b

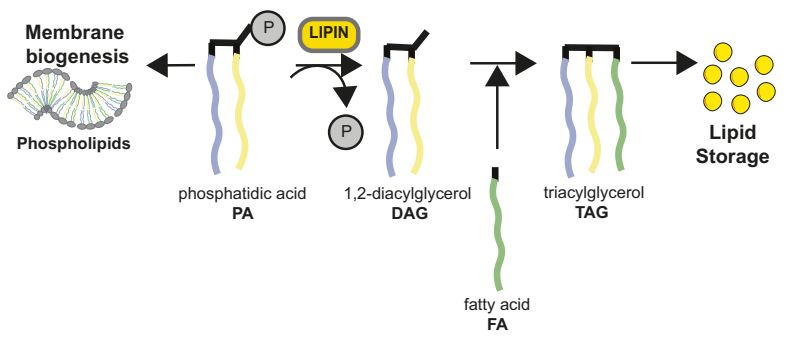

d

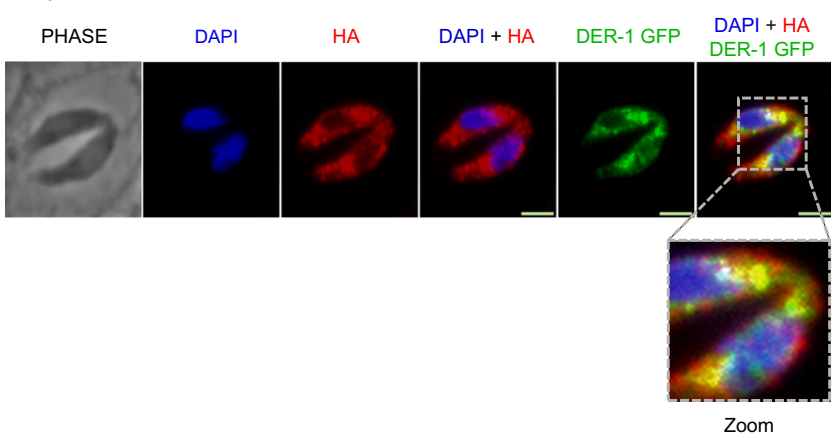

Fig. 1 T. gondii LIPIN (TgLIPIN) is a phosphatidate phosphatase localized to parasite cytoplasm and endoplasmic reticulum. a The C-LIP domain of TgLIPIN is evolutionarily conserved among eukaryotic orthologs and harbors the catalytic motif DVDGT known to be central to PA phosphatase activity. b Graphical scheme of the biochemical function of LIPIN in the glycerolipid biosynthesis pathway in eukaryotes. c The complementation of the yeast triple KO mutant ( $\triangle d p p 1 \Delta / p p 1 \Delta p a h 1)$, lacking phosphatidic acid phosphatase activity, with the C-LIP domain of TgLIPIN restores of PA and DAG ( $n=3$, unpaired $t$ test $P$ values where ${ }^{\star} P=0.024$ DAG triple mutant vs DAG complemented triple mutant, ${ }^{\star} P=0.032$ DAG triple mutant vs $D A G$ wild-type, ${ }^{\star}{ }^{\star} P=0.0039$ PA triple mutant vs PA complemented triple mutant, ${ }^{\star} P=0.039$ PA triple mutant vs PA wild type). Data are presented as mean values $+/-$ SEM. $\mathbf{d}$ IFA of TgLIPIN-HA (endogenous C-terminal tag) with anti-HA, DAPI, and a transiently expressed DER-1-GFP plasmid shows co-localization of TgLIPIN to the ER of the parasite. Scale bar: $2.0 \mu \mathrm{m}$.

suggests that TgLIPIN disruption dramatically impacts parasite intracellular growth and development TgLIPIN disruption caused a severe intracellular replication defect in $T g L I P I N-i k D$ with a significant increase of small vacuoles containing 1-2 parasite vacuoles (15-20\%) and vacuoles containing morphologically abnormal parasites (15-20\%), alongside with a concomitant significant decrease of larger vacuoles (25-30\%) containing 4-10 parasites (Fig. 2c). To further assess the effect of TgLIPIN on parasite intracellular growth, plaque assays were performed investigating the mutant's capacity to maintain proper growth with fluctuating levels of host nutrients. When cultured in regular growth conditions with $1 \%$ FBS, TgLIPIN-ikD (+ATc) exhibited a severe growth defect with few plaques (Fig. 2d, e) as expected from IFA results (Fig. 2b, f). To rescue this defect, the parasites were grown in high nutrient content with $10 \% \mathrm{FBS}^{4}$. However, surprisingly, TgLIPIN-ikD ( $+\mathrm{ATc}$ ) showed a more significant decline in growth in $10 \%$ than with $1 \%$ FBS containing culture medium, as marked by the complete absence of plaques (Fig. $2 \mathrm{~d}$, e). Contrastingly, a decrease in the host nutritional environment with $0 \%$ FBS slightly but significantly enhanced the growth of TgLIPIN-ikD (+ATc) (Fig. 2d, e) suggesting that exogenous lipid-nutrient source of FFA or PL are somehow toxic to parasites lacking TgLIPIN. To further delineate the division defect phenotype of TgLIPIN downregulation, we performed IFAs to probe the morphology of the inner membrane complex (IMC) as a marker of parasite shape and division. IFAs were conducted prior to and after complete protein loss, at $24 \mathrm{~h}$ (Fig. $2 \mathrm{f}$ and Supplementary Fig. 2c) and $48 \mathrm{~h}$ (Fig. $2 \mathrm{~g}$ ) of ATc treatment, respectively, under low, normal, and high host nutrient contents. Severe membrane anomalies and division defects were observed even as early as 24-h ATc treatment. Such membrane defects were strongly increased at 48-h ATc treatment, with membrane extrusion, loss of parasite integrity, and division arrest. Importantly, IMC and division defect were more evident when host nutrient content increased.

Electron microscopy reveals gross membrane anomalies as an early impact of $\mathbf{T g L I P I N}$ downregulation. To further resolve the cellular phenotype marked by TgLIPIN downregulation, TgLI$\mathrm{PIN}-\mathrm{ikD}(+\mathrm{ATc})$ parasites were examined by transmission electron microscopy (TEM) at 12,24 , and $48 \mathrm{~h}$ post ATc treatment under low, normal, and high host nutrient content $(0 \%, 1 \%$, and $10 \%$ FBS, respectively). Interestingly, as early as $12 \mathrm{~h}$ of treatment with ATc, when TgLIPIN levels are only slightly reduced, both parasite IMC and plasma membrane displayed gross abnormalities in forming evaginations or lateral interruptions, without affecting the parasite size (Fig. 3a-c). Concordantly, IFA data of TgLIPIN-ikD (+ATc) also hinted at aberrant IMC (Fig. 2f, g). The morphology of the nuclear envelope (NE) started to adopt tubular or multilobed shapes (Fig. $3 \mathrm{~d}-\mathrm{d}^{1}$ and Supplementary Fig. 3). Extensions of the NE seemed to expand in the cytoplasm in connection with the ER (Supplementary Fig. 3a) making tubular whorls in the cytoplasm (Fig. $3 \mathrm{e}-\mathrm{e}^{1}$ ). At 48 -h treatment, tubular or multilamellar membrane accumulation could entirely fill the cytoplasm of the parasites (Fig. $3 \mathrm{f}-\mathrm{f}^{1}$ ). Local fusion of the outer membrane of different nuclear lobes could be observed (Supplementary Fig. 3b). The NE tended to adopt hairpin shapes where the inner membrane from two different regions was seen closely apposed, separated by electron-dense material (Supplementary Fig. 3c, e). Local detachment and fusion of the inner membranes could be also observed (Supplementary Fig. 3e, red arrow). Interestingly, similar expansions affecting the NE are also 
a
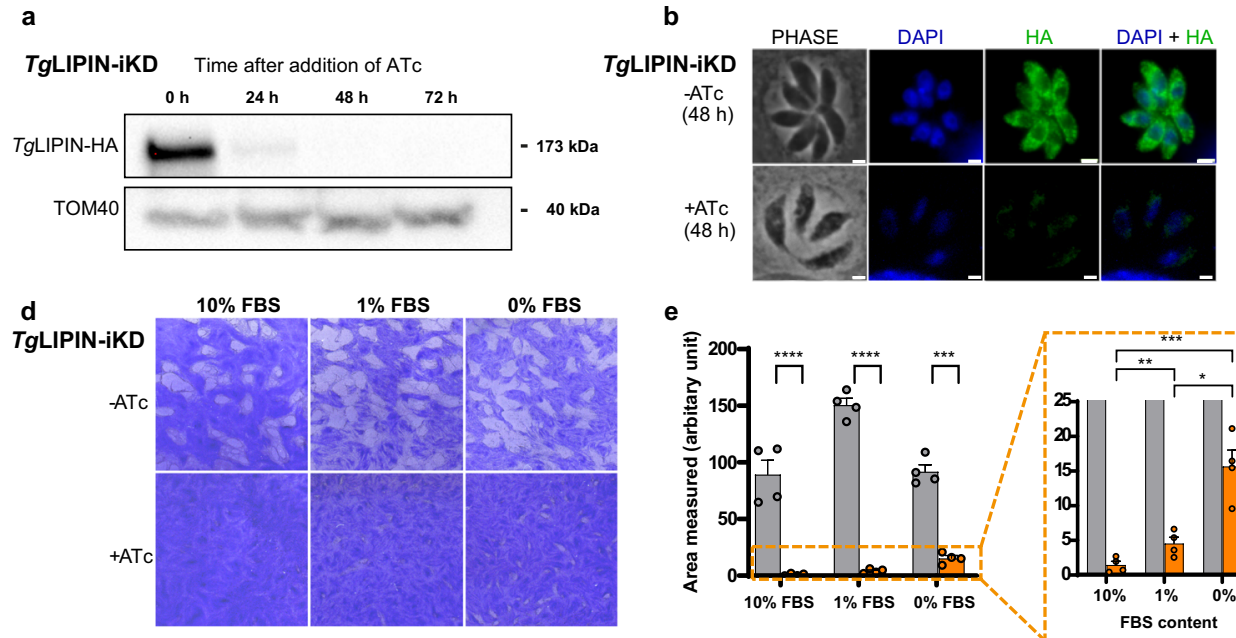

e
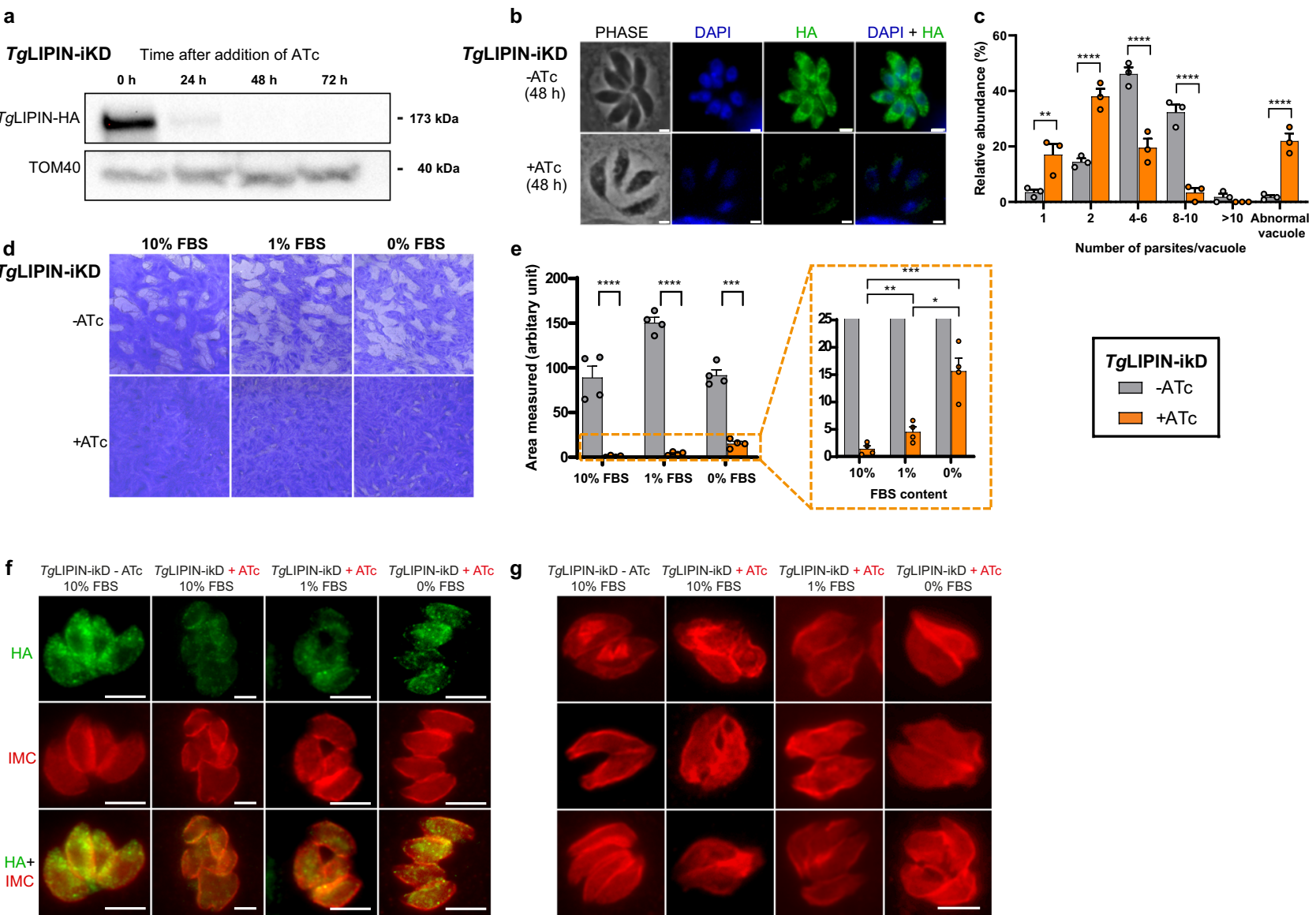

Fig. 2 TgLIPIN is indispensable for parasite replication and growth within its host. a Western blot shows TgLIPIN downregulation, $48 \mathrm{~h}+\mathrm{ATc}(0.5 \mu \mathrm{g} /$ $\mathrm{mL}$ ), TgLIPIN (anti-HA), and TOM40 (control). b IFA of TgLIPIN-ikD, indicating loss of protein using anti-HA antibody at $48 \mathrm{~h}+\mathrm{ATc}, \mathrm{Scale}$ bar: $2.0 \mu \mathrm{m}$. c Replication rate of TgLIPIN parasites grown with $(+)$ or without $(-)$ ATc measured by parasite number per parasitophorous vacuole after $24 \mathrm{~h}$ of growth post infection (>100 vacuoles were counted per biological replicate; $n=3$, unpaired $t$ test $P$ values where ** $P=0.002-\mathrm{ATc}$ vs $+\mathrm{ATc} 1$ parasite/vacuole, ${ }^{\star \star \star \star} P<0.000001-$ ATc vS + ATc 2/4-6/8-10 and abnormal parasite/vacuole). Data are presented as mean values $+/-$ SEM. d TgLIPIN-ikD plaque assays measuring parasite growth over 8-10 days ( $+/-\mathrm{ATC}$ ) in different FBS conditions. e shown as a bar graph (10\%, $1 \%$ and $0 \%$; $n=4$, unpaired $t$ test $P$ values where ${ }^{\star \star \star \star} P<0.000001-$ ATc vs + ATc 10/1/0\% FBS, ${ }^{\star \star} P=0.026+$ ATc $10 \%$ FBS vs + ATc $1 \%$ FBS, ${ }^{\star \star \star} p=0.0001+$ ATc $10 \%$ FBS vs + ATc $0 \%$ FBS, ${ }^{\star \star} P=0.0047+$ ATc $1 \%$ FBS vs + ATc $0 \%$ FBS). Data are presented as mean values $+/-$ SEM. f IFA illustrating early phenotypic effects of TgLIPIN depletion (24 h+ ATc) showing the presence of residual protein (HA-green). Inner membrane complex antibody (IMC- red) clearly shows aberrant IMC membrane biogenesis. $\mathbf{g}$ IFA illustrating the phenotypic effects of TgLIPIN depletion after $48 \mathrm{~h}(+\mathrm{ATC})$ using anti-IMC antibody in TgLIPIN-ikD. Scale bar: $5.0 \mu \mathrm{m}$.

observed in the yeast LIPIN mutant coupling phospholipid biosynthesis to the nuclear membrane $e^{25,26}$.

Both parasite IMC and plasma membrane (PM) also displayed abnormalities in forming bumps (Fig. 3d, red arrow) or lateral evaginations (Fig. $3 g-g^{2}$ ). Large inclusions could be observed in the vacuolar space that could correspond to such abnormal parasite evagination depending on the cutting plane. Complex structures were also observed in the cytoplasm, showing lasso-like structures formed by zippered membrane tubules (Supplementary Fig. 3f, g). It is not clear whether this type of structure originated from the ER, the nuclear membrane, or even IMC.

At $48 \mathrm{~h}$, the ultrastructure of the vacuole appeared dramatically altered. Endodyogeny was strongly affected in $T g L I P I N-i k D$ parasites, showing incomplete sister cells separation (Fig. $3 \mathrm{e}^{1}-\mathrm{f}^{\mathrm{l}}$ ), cytokinesis failure harboring multiple daughter cells (Fig. $3 \mathrm{~h}-\mathrm{i}^{2}$, red asterisk). Local fusion of the parasitophorous membrane (PVM) with the PM of the parasite was also observed (Fig. 3f). Some vacuoles showed signs of parasite PM rupture, with cytoplasmic material reminiscent of parasite cytoplasm filling the vacuolar space (Fig. $\left.3 \mathrm{~g}^{2}, \mathrm{~h}^{2}, \mathrm{i}^{1}\right)$. Large autophagosomes with unidentified content were also frequently observed in infected host cells suggesting that the parasites died and were eliminated by the host cell (Supplementary Fig. 3h-k). Large giant multivesicular bodies (gMVBs) and swelling of the host NE, previously described as enhanced under starvation conditions ${ }^{4}$ were abundant in TgLIPIN-ikD parasites, even in the presence of $10 \%$ FBS. In some cases, the outer membrane of the NE had fused with the PVM (Supplementary Fig. 3c), occasionally leading to its rupture (Supplementary Fig. 3d).

In agreement with the replication assay data (Fig. 2c), TEM images showed that dynamics of parasite membranes was profoundly affected by the absence of appropriate levels of TgLIPIN, which seemed to start at the NE level as soon as $12 \mathrm{~h}$ of treatment. Further loss of TgLIPIN then induced strong aberrant membrane abnormalities mostly affecting the endomembrane system (NE, ER, IMC, and PM). This resulted in endodyogeny failure, anarchic membrane proliferation, and unexpected membrane fusion events, leading to parasite growth arrest and death. These membrane abnormalities and division phenotypes were observed in all host nutrient contents, but were always 

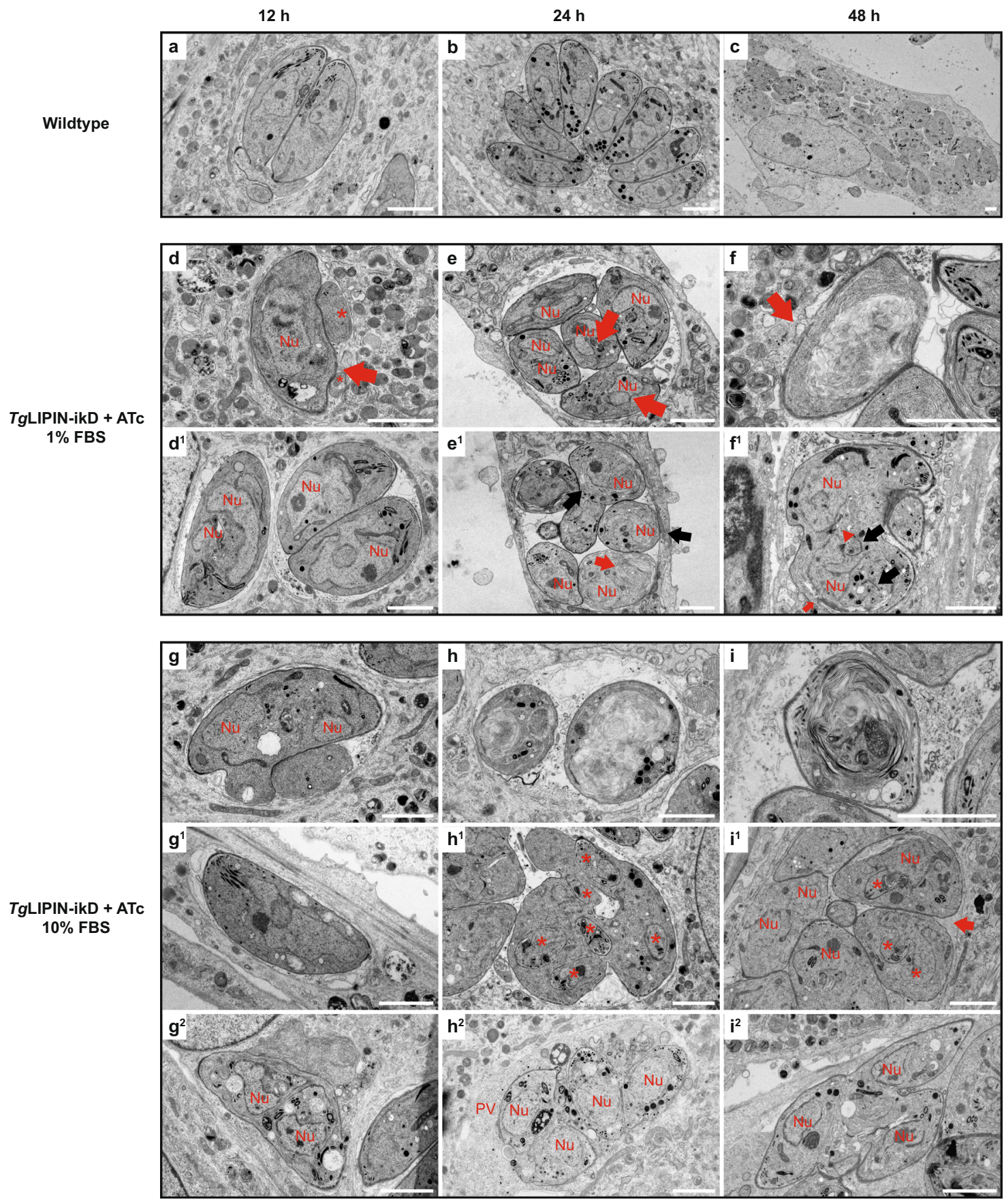

Fig. 3 TgLIPIN depletion results in gross membrane anomalies early in the process of ATc downregulation. Transmission electron micrographs showing wild-type (a-c) and TgLIPIN-ikD parasites at 1\% (d-f) or 10\% FBS (g-i), after 12, 24, and $48 \mathrm{~h}$ of ATc treatment. After $12 \mathrm{~h}$ of growth, wild-type parasites showed vacuoles containing (a) 2-4 parasites, (b) 8-16 parasites at 24h, and (c) after $48 \mathrm{~h}$, the first round of egress/reinvasion has occurred showing cells with multiple small vacuoles showing regular nuclei and organelles. $\mathbf{d}-\mathbf{f}$ TgLIPIN-ikD parasites show membrane anomalies as early as $\mathbf{d}-\mathbf{d}^{\mathbf{1}} 12 \mathrm{~h}$ of induction. Vacuoles showed large inclusions in the parasitophorous vacuole, red asterisks. $\mathbf{d}^{\mathbf{1}}$ Nuclei showed a multilobed shape even in the absence of

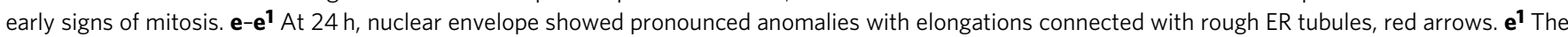
ER showed exaggerated proliferation generating big membrane whorls. Incomplete cytokinesis was observed, black arrow. f Forty-eight hours after induction, parasites were full of ER-derived membranes and there was the fusion of the parasitophorous membrane (PVM) and parasite plasma membrane (PM), red arrow. $\mathbf{f}^{\mathbf{1}}$ Endodyogeny defect: a new round of mitosis started, shown by the presence of IMC buds (black arrows) and centrocone (red arrowhead), while two sister cells from the previous generation have not separated. $\mathbf{g}-\mathbf{i}$ All membrane defects were present and enhanced in $10 \%$ FBS. $\mathbf{g}-\mathbf{g}^{\mathbf{1}}$ At $12 \mathbf{h}$, nucleus elongation and cytoplasmic evaginations were observed. $\mathbf{g}^{\mathbf{2}}$ Cytokinesis failure was observed. $\mathbf{h}-\mathbf{h}^{\mathbf{2}}$ At 24 h, ER proliferation and cytokinesis defect were observed. Vacuoles showed signs of necrosis with PM rupture and cytoplasmic leakage in the vacuolar space. i At $48 \mathrm{~h}, T g L I P I N$-ikD did not reinvade, the remaining vacuoles showed profoundly abnormal parasites, $\mathbf{i}^{\mathbf{1}}$ PVM or $\mathbf{i}^{\mathbf{2}}$ PM rupture/cytokinesis failure. Nu nucleus. Scale bar: $2 \mu \mathrm{m}, n=3$. 
stronger and aggravated under high host nutrient content $(10 \%$ FBS). This suggests that the essential role of TgLIPIN for membrane biogenesis and parasite survival is directly linked to the nutritional status of the host.

TgLIPIN regulates lipid homeostasis. To determine the functional role of TgLIPIN in parasite PA synthesis and membrane biogenesis, we conducted comprehensive lipidomic analyses. To precisely assess the timely impact of TgLIPIN-ikD disruption on lipid synthesis, parasites were grown for 24 or $48 \mathrm{~h}$ with and without ATc. Total lipids were separated by high-performance thin-layer chromatography (HPTLC) and quantified by gas chromatography-mass spectrometry (GC-MS). Relative abundance of PA was unchanged at $24 \mathrm{~h}$ of + ATc (Fig. $4 \mathrm{a}$ ). After $48 \mathrm{~h}$ $+\mathrm{ATc}$, there was a large significant increase of PA, confirming its role for PA synthesis and our WB results for the loss of TgLipin (Fig. 4a). FA composition of PA showed an increase in the level of myristic (C14:0) and palmitic (C16:0) acids along with a decline in the levels of stearate (C18:0) (Fig. 4b). Concomitantly to PA increase, the relative abundance of DAG was decreased after $48 \mathrm{~h}$ of TgLIPIN downregulation by ATc (Fig. 4c) showing that TgLIPIN is an active phosphatidic acid phosphatase, controlling the levels of PA and DAG, as confirmed by our yeast heterologous complementation (Fig. 1c). Despite a strong replication defect and reduction in parasite number, the total amount of phospholipids was increased up to 1.5 -fold in the TgLIPIN-ikD $(+\mathrm{ATc})$ in comparison to the control (-ATc) (Supplementary Fig. 4a). Accordingly, most PL classes were further increased by TgLIPIN disruption such as the ones usually made from PA via the CDP-DAG pathway ${ }^{25}$ i.e., cardiolipin (CL), PS, and PC (Supplementary Fig. 4b). This suggests that TgLIPIN controls PL homeostasis likely through PA synthesis.

In eukaryotes, TAG biosynthesis occurs at the last step of the glycerol-3-phosphate pathway through the $s n-3$ acylation of DAG catalyzed by a diacylglycerol-acyltransferase (DGAT) that uses $\mathrm{FFA}^{7,26,27}$. However, the key and limiting step of TAG synthesis is the formation of its precursor, DAG, the production of which depends on the action of a PAP/LIPIN. TAG is not a membrane but a storage lipid that accumulates within lipid droplets together with other neutral lipids such as cholesteryl esters (CE). We thus investigated the putative role of TgLIPIN in lipid-storage formation by comparing the number of parasite lipid droplets using nile red staining ${ }^{28}$ in the TgLIPIN-ikD parasites ( + ATc and $-\mathrm{ATc}$ ). This showed that the number of lipid droplets per parasite vacuole in the presence of ATc was reduced to almost $60 \%$ in comparison to the control in both normal (1\% FBS) and furthermore in high host nutrient content (10\% FBS) (Fig. $4 \mathrm{~d}$, e), suggesting that $\operatorname{TgLIPIN}$ is involved in the synthesis of storage lipids. According to this hypothesis, the relative abundance of TAGs was significantly reduced after $48 \mathrm{~h}$ of ATc treatment (Fig. 4f). Other neutral lipids making the bulk of lipid droplets, CE levels were also significantly decreased (Supplementary Fig. 4d).

TAGs and CEs are neutral storage lipids potentially implicit in the parasite's ability to cope with the excess FA? ${ }^{7}$. The synthesis of TAG via enzyme DGAT requires two substrates, DAG and FFA. We, therefore, determined the FFA content of the TgLIPIN-ikD. Lipidomics revealed slight increases in total FFA levels in the TgLIPIN-ikD (+ATc/-ATc) (Fig. 4g). Interestingly, C18:1 (oleate) was the only FA found significantly increased in the parasite-free FA (FFA) pool (Supplementary Fig. 4c), suggesting C18:1 may be an important intermediate in the pathways regulated by $T g L I P I N$, as previously hinted ${ }^{7,29,30}$. In a high host environment (10\% FBS), lipidomic analyses revealed that only TAG levels decreased under these conditions (Fig. 4h), explaining the reduction of lipid droplets. This impairment in TAG biosynthesis is consistent with the reduction in parasite viability seen under high FBS conditions (Fig. 2d, e).

TgLIPIN channels the flux of host fatty acids to prevent the accumulation of free fatty acids levels and promotes TAG synthesis. Our data reveal the essential role for TgLIPIN in regulating PA and DAG synthesis to control lipid homeostasis, especially the level of storage lipid molecule, TAG. TgLIPIN function seems directly connected to host nutritional status and the origin of lipid precursors used (FASII, host cell, and external environment) to maintain parasite survival.

Fatty acids required for lipid synthesis in T. gondii parasites are obtained from the host and/or host environment (i.e., medium) $)^{1,2,4,26,29}$ and/or synthesized de novo via the apicoplast FASII pathway ${ }^{12,30,31}$. To identify the source of increased FA and phospholipids detected upon TgLIPIN depletion, we set up fluxomics approaches where we grew parasites and/or host cells using different stable isotope substrates-containing media $\left({ }^{13} \mathrm{C}\right.$ $\mathrm{U}$-glucose, $\left.{ }^{31} \mathrm{~d}-\mathrm{C} 16: 0\right)$ and performed lipidomic analyses for each experimental setup (Fig. $5 \mathrm{a}-\mathrm{c}$ ).

To monitor apicoplast FASII activity, parasites were labeled with $\mathrm{U}^{13} \mathrm{C}$-glucose, which was added to a glucose-free medium, to confluent host cells together with parasites with or without ATc (Fig. 5a), as previously reported ${ }^{13}$. Total lipid content, PL, and neutral lipids were extracted to determine the ratio of ${ }^{13} \mathrm{C}$ incorporation of each FA as a measure of FASII activity. In TgLIPIN-ikD ( $+\mathrm{ATc})$, the amount of labeled FA made by FASII (i.e., FASII activity) decreased under the suppression of TgLIPIN (+ATc) in terms of total lipid content (Fig. 5d) as well as the pools of FFA/PL/TAG (Fig. 5e, f and Supplementary Fig. 5a, b). Interestingly, there was a significant decrease in all the signature FA from FASII (i.e., C14:0) but also in FASII-derived FA, i.e., oleic acid C18:1, reduced in total lipids, FFA, PL and TAG made from FASII (Fig. 5d, e, f and Supplementary Fig. 5a, b). There was an overall decrease in FASII-derived C18:1 in FFA (Fig. 5e) and TAG (Fig. 5f, supporting the lipid droplet analyses) and an increase in PC16:0 (Supplementary Fig. 5b). This suggests that with the loss of TgLIPIN, the increased overall PA levels with FASII-derived FAs, can be directed towards the production of excess PC. The distribution of ${ }^{13} \mathrm{C}$ incorporation to each isotopologue of FASII FA product confirmed that the pathway was nevertheless functional (Supplementary Fig. 5c-g). To monitor the FAs sourced from the external environment/culture medium, we grew parasites and confluent host cells in a medium supplemented with deuterated palmitic acid ( $\left.{ }^{31} \mathrm{~d}-\mathrm{C} 16: 0\right)$ (Fig. 5b). Parasites were harvested and total lipids were extracted to determine the incorporation of ${ }^{31} \mathrm{~d}-\mathrm{C} 16: 0$ in parasite lipids. In the total lipid content, ${ }^{31} \mathrm{~d}-\mathrm{C} 16: 0$ and its elongation/desaturation products ${ }^{31} \mathrm{~d}-\mathrm{C} 18: 0$ and ${ }^{29} \mathrm{~d}-\mathrm{C} 18: 1$ were detected in lower amounts in the TgLIPIN-ikD parasites with ATc (Fig. 5g). In addition, a drastic reduction in the incorporation of all three ${ }^{31} \mathrm{~d}$ C16:0, $\left.{ }^{31} \mathrm{~d}-\mathrm{C} 18: 0,{ }^{29} \mathrm{~d}-\mathrm{C} 18: 1\right)$ to FFA species within TgLIPIN-ikD (+ATc) in comparison to the control (-ATc), further suggesting that the host external environment is not the source of increased lipids within the mutant (Fig. 5h).

To monitor the fatty acids directly scavenged from the host, we designed a novel assay using $\mathrm{U}^{13} \mathrm{C}$-glucose to label host lipids (Fig. 5c). Nonconfluent host cells were grown in the presence of medium containing $\mathrm{U}-{ }^{13} \mathrm{C}$-glucose which would theoretically fuel any of the active FA synthesis pathways within the host (FASI, elongases) via the synthesis of their substrates, acetyl-CoA, thereby generating ${ }^{13} \mathrm{C}$-pre-labeled host metabolites including lipids and fatty acids ${ }^{32}$. These ${ }^{13} \mathrm{C}$-pre-labeled host cells were then infected with parasites in the presence of a normal culture medium, which contains regular ${ }^{12} \mathrm{C}$-glucose. Total lipid was 
a

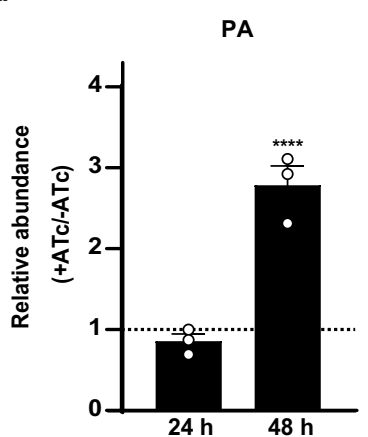

b

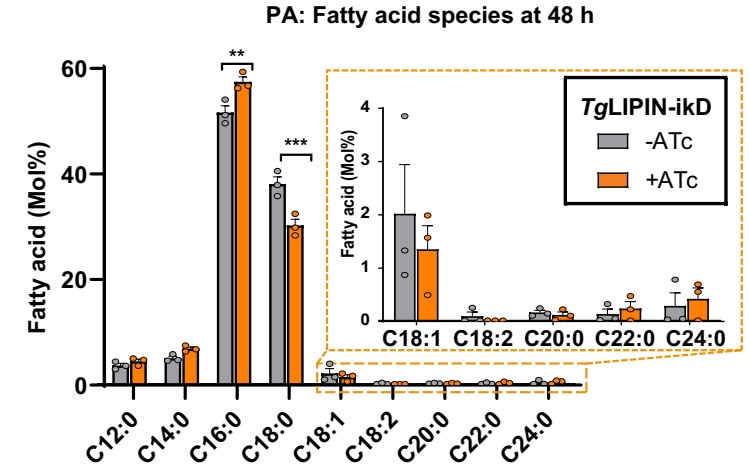

c

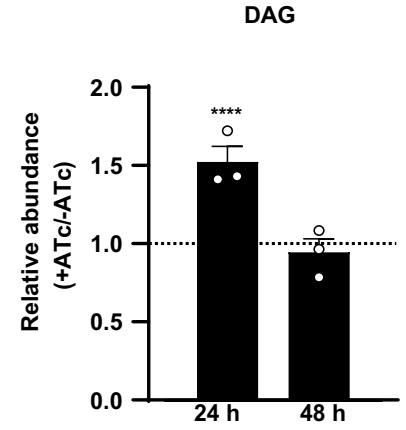

d

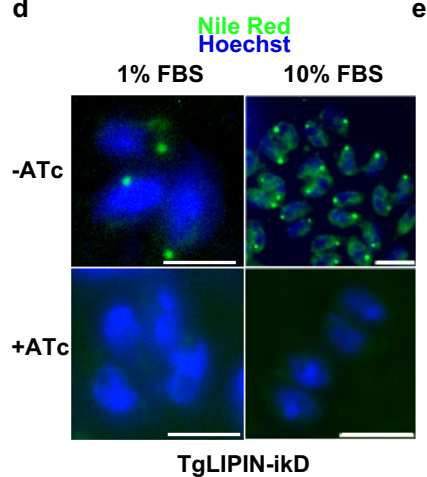

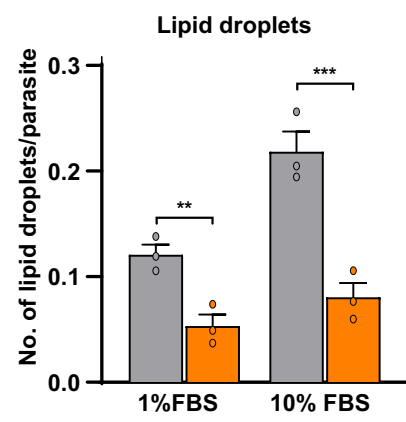

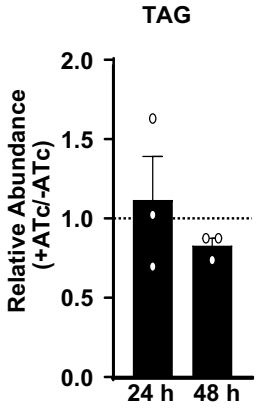

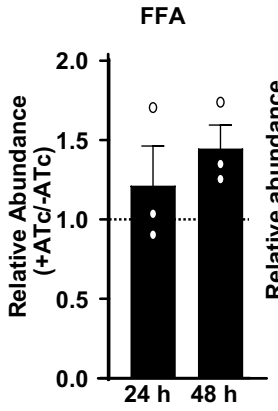

h

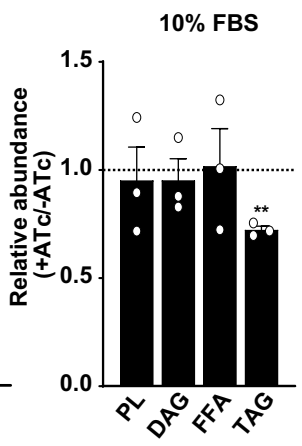

Fig. 4 TgLIPIN regulates critical levels of PA and other major phospholipids while generating DAG that is directed toward neutral lipid storage. a Relative abundance of PA in TgLIPIN-ikD (nmol/parasites number, $+\mathrm{ATc} /-\mathrm{ATc} ; \% ; n=3$, unpaired $t$ test $P$ values where ${ }^{\star \star \star \star} P<0.00001$ relative PA abundance ratio nmol/parasites number $+A T c /-A T c$ at $48 \mathrm{~h}$ vs + ATc/ $-A T c=1)$. b Fatty acid composition of PA in TgLIPIN-ikD $(+/-A T c)$ in Mol\%; $n=3$, unpaired $t$ test $P$ values where ${ }^{\star \star} P<0.0021$ PA C16:0 - ATc vs + ATc, ${ }^{\star \star \star} P<0.0002$ PA C18:0 - ATc vs + ATc. c Relative abundance of DAG $(+\mathrm{ATC} /-\mathrm{ATC})$ is reduced by $T g$ LIPIN depletion after $48 \mathrm{~h}+\mathrm{ATC}\left(n=3\right.$, unpaired $t$ test $P$ values where ${ }^{\star \star \star \star} P<0.00001$ relative DAG abundance ratio $\mathrm{nmol} /$ parasites number $+\mathrm{ATc} /-\mathrm{ATc}$ at $24 \mathrm{~h}$ vs $+\mathrm{ATc} /-\mathrm{ATc}=1)$. $\mathbf{d}$ Nile red staining showed the number of lipid droplet within the parasites after $24 \mathrm{~h}$ of $+/-$ ATc $($ scale bar $=5.0 \mu \mathrm{m})$ with $(\mathbf{e})$ a representative bar graph $\left(n=3\right.$, unpaired $t$ test $P$ values where ${ }^{\star} P<0.0093$ number of lipid droplets per parasite + ATc/ - ATc at $1 \%$ FBS, ${ }^{\star \star \star} P<0.0003$ at $10 \%$ FBS). $\mathbf{f}$ Relative abundance of TAGs in TgLIPIN-ikD ( + ATc/ - ATc) showing that TAG levels were relatively reduced in the TgLIPIN-ikD ( $48 \mathrm{~h}+\mathrm{ATc})$. $\mathbf{g}$ Relative abundance of free fatty acids (FFA, + ATc/ - ATc in the TgLIPIN-ikD). $\mathbf{h}$ Relative abundance of PLs, DAG, FFA and TAG in the TgLIPIN-ikD +ATc to - ATc at $10 \%$ FBS $\left(n=3\right.$, unpaired $t$ test $P$ values where ${ }^{\star \star} P<0.004$ relative TAG abundance ratio nmol/ parasites number $+\mathrm{ATc} /-\mathrm{ATc}$ at $10 \% \mathrm{FBS}$ vs $+\mathrm{ATc} /-\mathrm{ATc}=1$ ). Data are presented as mean values $+/-\mathrm{SEM}$.

extracted from the parasites to determine the ratio of ${ }^{13} \mathrm{C}$ incorporation to each fatty acid and determine the origin from the host or not. Using this novel approach, we were able to determine (i) the FA biosynthetic capacities of the host cells (Fig. 5i), and more importantly (ii) the first scavenged FA lipidome of the parasite (Fig. $5 \mathrm{j}-\mathrm{m}$ ), the scavengome. Host cells, human foreskin fibroblast (HFF), are capable of synthesizing FA ranging from $\mathrm{C} 14: 0$ to $\mathrm{C} 20: 1$, with the most abundant FA species being C18:1 (Fig. 5i). The wild type (TgLIPIN-ikD -ATc) parasites are capable of scavenging all these FA species made by the host cell with a major preference again for C18:1 (Fig. 5j). Importantly, in TgLIPIN mutant $(+\mathrm{ATc}),{ }^{13} \mathrm{C}$-labeled C16:0, C18:0 and C18:1 from host cells were significantly increased in the FFAs (Fig. 5k), together with a significant increase of hostderived C18:0 in both parasite total lipids and PL (Fig. 5j, 1). Therefore, since FA from both FASII and the external environment decrease in the absence of TgLIPIN, whilst FAs from host cell are found increasing, our data strongly suggest that the major origin of excess lipids in the TgLIPIN mutant is from the host cell. Correlating with the lipid droplet analyses, there was a significant decrease in the ${ }^{13} \mathrm{C}$ labeled FA, specifically C16:1 and C18:1, in TAG (Fig. $5 \mathrm{~m}$ ). The distribution of ${ }^{13} \mathrm{C}$ incorporation to each isotopologue of host-derived FAs was also determined
(Supplementary Fig. 5h-m). Together these data show that the disruption of TgLIPIN induced the massive accumulation of host cell-scavenged FA, specifically including C18:1, that cannot be used for TAG synthesis.

Hence, TgLIPIN regulates parasite membrane biogenesis by controlling the synthesis and content of PA and DAG, and by channeling the constant flux of host cell scavenged FA for TAG. Without TgLIPIN, the parasite accumulates toxic levels of PA, FFA, and PL that eventually cause massive membrane malformations, cytokinesis defect, and parasite death.

\section{Discussion}

TgLIPIN plays a pivotal role in the synthesis of central lipid precursors, PA and DAG while regulating the flux of host lipids thereby controlling the equilibrium between membrane biogenesis and lipid storage. Collectively this controls intracellular development and the propagation of the parasite (Fig. 6a).

Our data here show that TgLIPIN is an essential PAP controlling the PA/DAG equilibrium along with the level of FFAs. Disruption of this equilibrium together with impairment of storage capacity has multiple phenotypic consequences within the parasite (Fig. 6b). In mammalian cells, LIPIN is encoded by three 
a

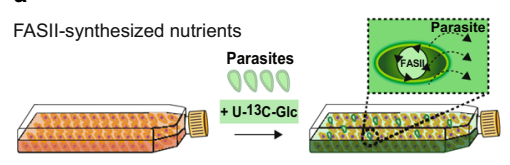

b

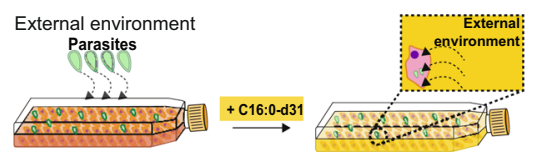

c

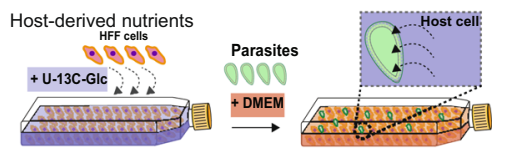

d

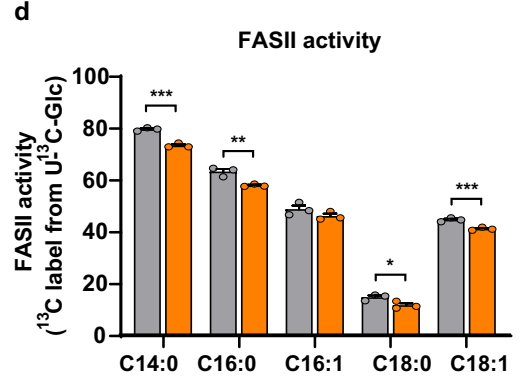

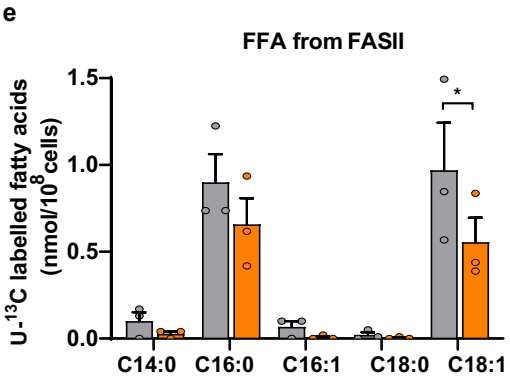

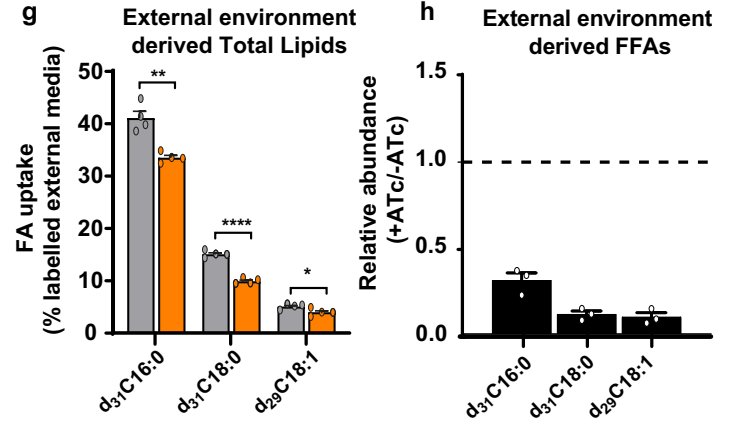

k

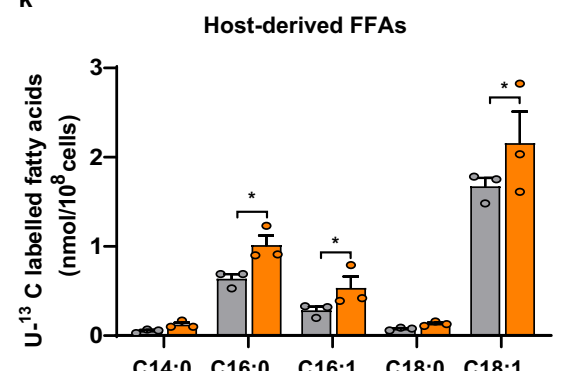

I

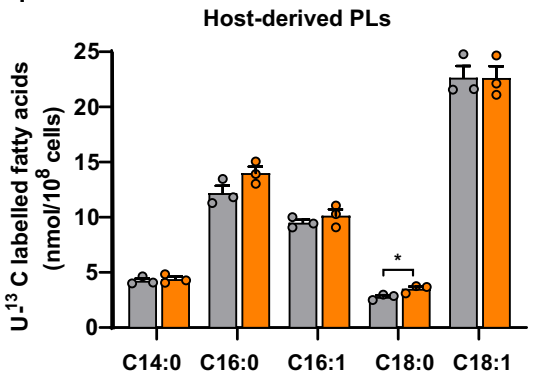

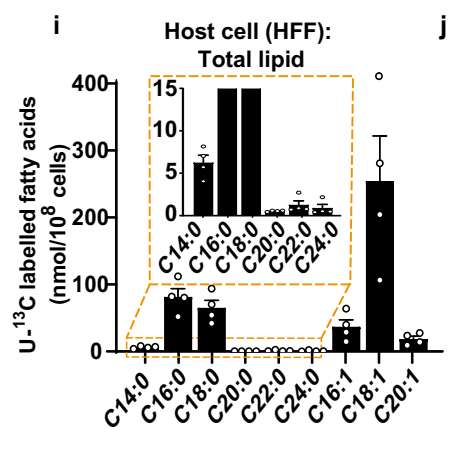

j f

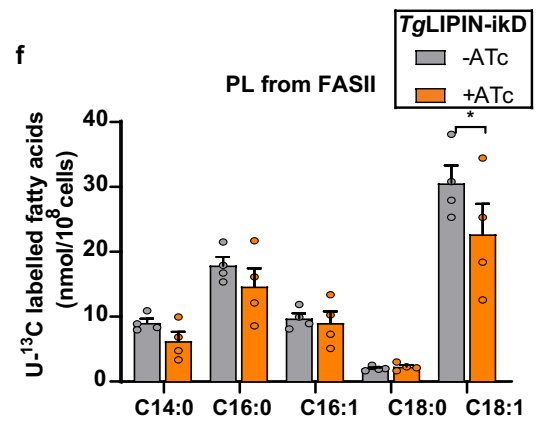

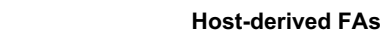

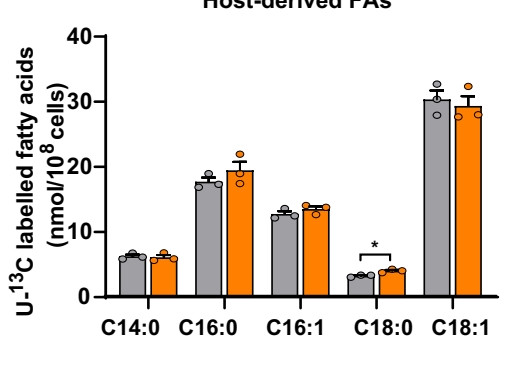

m

Host-derived PLs

Host-derived TAG

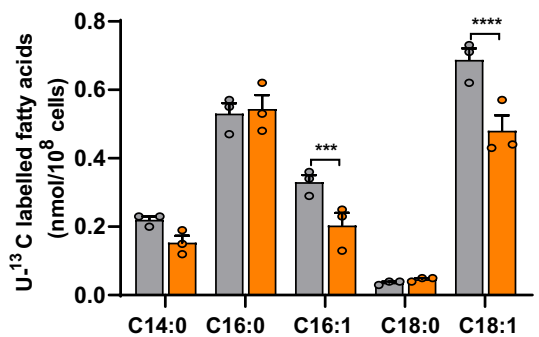

Fig. 5 Monitoring the source of fatty acids in TgLIPIN-ikD. a-c Schematic for the experimental procedure of stable isotope labeling to determine the origin of fatty acids in TgLIPIN-ikD; a Parasite FASII derived FAs were determined by the addition of U-13 C-glucose (Glc) to the culture medium (1\% FBS, no glucose, $+/-$ ATc) at the time of parasite infection to the host. $\mathbf{b}$ FAs scavenged from the external environment (medium) was determined by the addition of ${ }^{31} \mathrm{~d}$-palmitic acid $\left({ }^{31} \mathrm{~d}-\mathrm{C} 16: 0\right)$ to the parasite culture medium (high glucose, 1\% FBS). c Host cell-derived FAs were determined by growing host cells in the presence of $\mathrm{U}-{ }^{13} \mathrm{C}$-glucose (no glucose, 10\% FBS) to confluent in prior to the infection parasites with normal medium (glucose, $1 \% \mathrm{FBS},+/-\mathrm{ATC}$ ). d FASII activity in TgLIPIN-ikD as measured as $\%{ }^{13} \mathrm{C}$-incorporation $\left(n=3\right.$, unpaired $t$ test $P$ values; ${ }^{\star \star \star} P<0.0005 \%$ FASII derived C14:0/C16:0/C18:0/ C18:1 - ATC vs + ATc, $\left.{ }^{\star \star} P<0.007 /{ }^{\star} P<0.04 /{ }^{\star \star \star} P<0.0003\right)$. e Abundance of apicoplast FASIl derived ${ }^{13} \mathrm{C}$ labeled FFAs $(n=3$, unpaired $t$ test $P$ values; ${ }^{\star} P<0.02$ of FASII derived FFA C18:1-ATc vs + ATC). $\mathbf{f}$ Abundance of apicoplast FASII derived ${ }^{13}$ C-labeled phospholipids (PLs, $n=4$, unpaired $t$ test $P$ values; ${ }^{\star} P<0.03$ of FASII derived PL C18:0-ATc vs + ATc). $g$ FA uptake of deuterated external environment-derived FAs ( $n=4$, unpaired $t$ test $P$ values; ${ }^{\star \star} P=0.0034 /{ }^{\star \star \star} P<0.00001 /{ }^{\star} P=0.01 \mathrm{FA}$ uptake from the external environment as $\mathrm{d}_{31} \mathrm{C} 16: 0 / \mathrm{d}_{31} \mathrm{C} 18: 0 / \mathrm{d}_{29} \mathrm{C} 18: 1-\mathrm{ATc}$ vs $\left.+\mathrm{ATC}\right)$. $\mathbf{h}$ Relative abundance of deuterated external environment-derived ( + ATc/-ATc) $(n=3)$. $\mathbf{i}$ The abundance of ${ }^{13} \mathrm{C}$-labeled fatty acids in the host cells $(n=4)$. $\mathbf{j}$ Host derived FAs measured as $\%{ }^{13} \mathrm{C}$-incorporation ( $n=3$, unpaired $t$ test $P$ values; ${ }^{\star} P<0.02$ Host derived FA C18:1-ATc vs + ATc). $\mathbf{k}$ Abundance of host-derived ${ }^{13} \mathrm{C}$-labeled FFAs $\left(n=3\right.$, unpaired $t$ test $P$ values; ${ }^{\star} P<0.05$ Host derived FA C16:0/16:1/18:1-ATc vs + ATc). I Abundance of host-derived ${ }^{13} \mathrm{C}$ labeled PL $(n=3$, unpaired $t$ test $P$ values; ${ }^{\star} P<0.023$ Host derived PL C18:0-ATc vs + ATc $)$ and $\mathbf{m}$ TAG levels $\left(n=3\right.$, unpaired $t$ test $P$ values; ${ }^{\star \star \star} P<0.0001$ Host-derived TAG C16:0/18:1-ATc vs +ATC). Data are all presented as mean values + /- SEM.

independent genes lipin1, 2, 3; all of which have PAP activity modulating the levels of TAGs and phospholipids ${ }^{21}$. Genetic mutation within these LIPINs causes severe metabolic disorders, including rhabdomyolysis (lipin1), obesity (lipin1), autoinflammatory disease (lipin2), and impaired lipoprotein assembly in the intestines (lipin 2 and 3$)^{33}$. The essentiality of LIPIN for $T$. gondii growth also suggests distinct functionality from the two other parasite PAPs. Neither Toxoplasma PAPs appeared to compensate for the loss of TgLIPIN, unlike in yeast whereby deletion of genes encoding LIPIN can be partly compensated by other PAPs ${ }^{19}$. The absence of a NLS (nuclear localization signal) in the TgLIPIN sequence is consistent with a cytosolic localization, colocalization specifically with the NE membrane, ER, and other endomembrane. The localization and catalytic activity of 


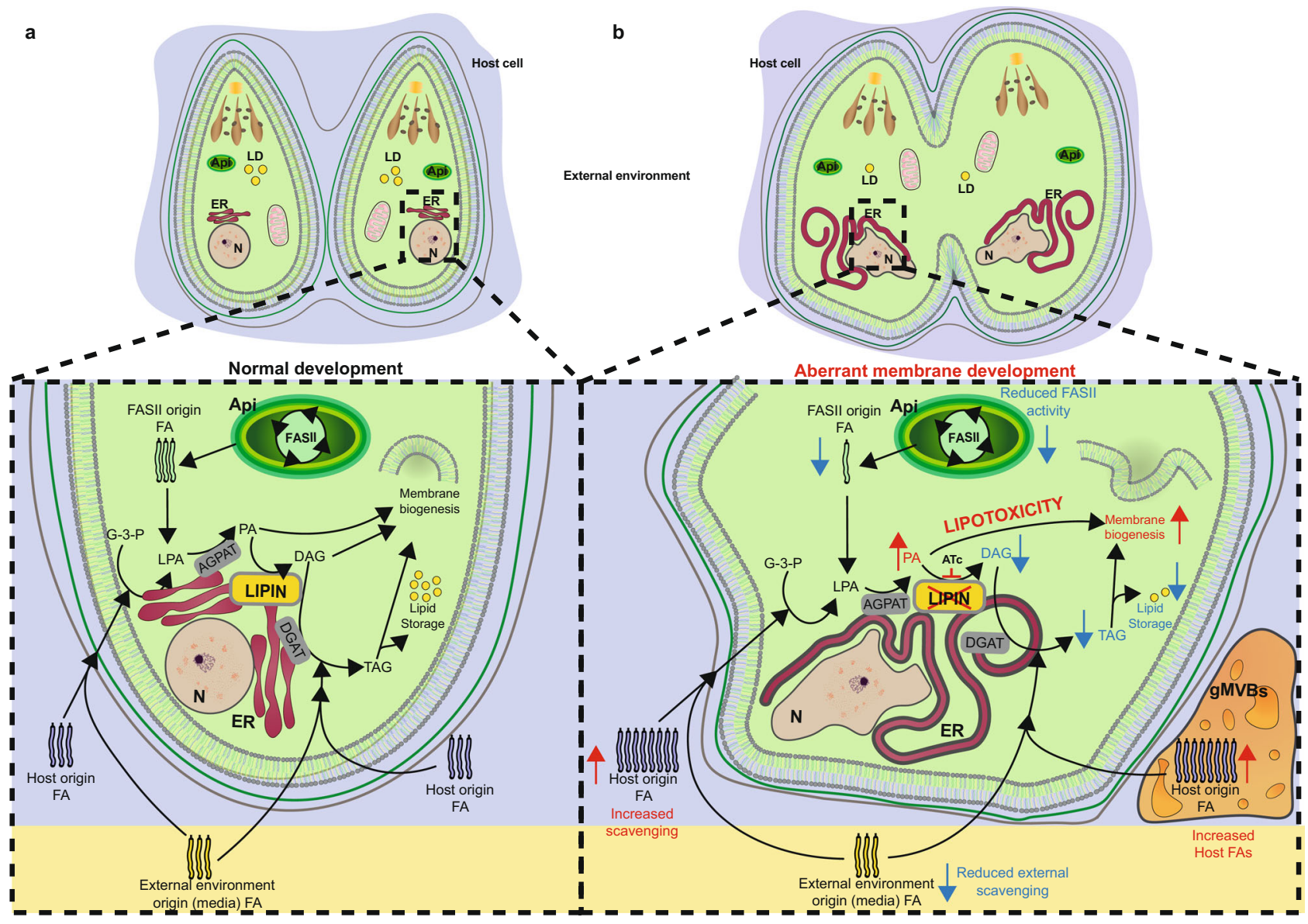

Fig. 6 Proposed role of LIPIN in Toxoplasma lipid metabolism. Schematic representation of the dual essential role of TgLIPIN in parasite lipid metabolism, including, membrane biogenesis, and lipid storage. a The parasite utilizes, and glycerol-3-phosphate (G-3-P) and fatty acid (FA)s derived from both apicoplast (Api) FASII and host to synthesize major lipids by forming lysophosphatidic acid (LPA) followed by phosphatidic acid (PA). PA is hydrolyzed by TgLIPIN to generate diacylglycerol (DAG), which is further acylated to generate triacylglycerol (TAG). The other branch of this pathway redirects PA toward the generation of major membrane phospholipids. The FA homeostasis between membrane biogenesis and storage essential for normal parasite development within its host is maintained by the PA phosphatase TgLIPIN. b Genetic ablation of TgLIPIN in TgLIPIN-ikD parasites in the presence of anhydrotetracycline (ATc) caused the PA/DAG imbalance. The increased PA is channeled towards PL synthesis resulting in excess PLS and consequent gross membrane anomalies within the parasite IMC, nucleus, and ER, while forming giant multivesicular bodies ( $g M V B s$ ). Simultaneously, the reduction in DAG affected TAG biogenesis and hence the lipid storage capacity, marked by decreased lipid droplets (LD), within the parasite lacking TgLIPIN. The impairment of TAG biosynthesis, resulted in excess fatty acids derived from the host, within TgLIPIN mutant, causing lipotoxicity.

LIPIN proteins in humans and yeast are regulated by a phosphatase bearing the HAD domain as its substrate ${ }^{34-36}$.

Therefore, it is conceivable that TgLIPIN is quite dynamic and can be recruited on endosomal membranes to regulate the PA/ DAG equilibrium, depending on metabolic demand, cell cycle, or coupled to membrane trafficking events. This hints at a probable link between metabolism and the nucleus of the parasite, however contrary to yeast and mammals, there is no evidence of a direct effect of TgLIPIN on gene regulation, which could explain the absence of nuclear localization.

One of the early phenotypic stresses evoked in the TgLIPINdeficient mutant was the impact on major membranes including the nuclear envelope complexed with the ER and the parasite IMC. There is evidence that suggests that nuclear membrane biogenesis is linked to PA metabolism ${ }^{37,38}$. In $\Delta p a h 1$ yeast, mutations in upstream biosynthetic steps of the glycerolipid pathway that lower PA levels also reduced the aberrant expansion of nuclear membrane ${ }^{37}$. Effects of pah1 deletion could be phenocopied by overexpression of Dgk1p, a nuclear/ER membrane DAG kinase that generates $\mathrm{PA}^{37}$.
A recent study demonstrated that the use of DGAT inhibitorT863, inhibiting DAG to TAG synthetic reaction in T. gondii parasites resulted in rapid accumulation of membranous structures accompanied by rapid parasite death due to strong replication defect ${ }^{6}$. This cytopathy was restricted mainly to the ER and the associated nuclear envelope, similarly as in the case of TgLIPIN depletion. The electron micrographs of the TgLIPIN mutant $(+\mathrm{ATc})$ clearly suggest that the replication arrest was initially due to profound modifications of the nuclear membrane resulting in aberrant membrane proliferation, cytokinesis defect, and parasite death.

In the absence of TgLIPIN, we observed an overall increase in the major membrane phospholipids, including CL, PS, and PC which is a derivative of the CDP-DAG pathway in other eukaryotes $^{39}$. In absence of DAG, PL biosynthesis was expected to be reduced. Instead, an increase in both PL and FFA is observed, which is likely due to excessive host lipid scavenging. The TAG content in TgLIPIN mutant decreases only $48 \mathrm{~h}$ post protein downregulation with ATc suggesting that due to a decrease in the substrate-specific DAG species because of TgLIPIN unavailability, 
the parasite is unable to acylate the free fatty acids to generate its subsequent product TAG. This is supported by the drop in lipid droplet numbers in $1 \%$ and $10 \%$ FBS when $T g$ LIPIN is suppressed and the exaggerated effect in a high host nutrient environment (Fig. 4d, e). In mammalian cells the inhibition of TAG synthesis leads to oleic acid (C18:1) induced lipotoxicity ${ }^{39}$. Thus, lipid droplets act as more than just cellular energy reserves by protecting the cells from deleterious effects of toxic excess FFAs by their incorporation into TAGs.

The prelabeling of host fibroblast cells with $\mathrm{U}^{13} \mathrm{C}-\mathrm{Glucose}$, allowed us to track the source of excess fatty acid and phospholipid content in the TgLIPIN mutant. In keeping with previously reported data, using ${ }^{13} \mathrm{C}$-fluxomics we observed that the parasite has a specific preference for scavenging oleate (C18:1) directly from host ${ }^{40}$. Existing literature provides strong evidence of the involvement of C18:1 in TAG biosynthesis and subsequent lipid droplet formation in mammals ${ }^{39}$ as well as parasites ${ }^{7}$. Thus, the excess oleate in the form of FFAs within the parasite sourced directly from the host has a strong correlation to the lipid storage defect in TgLIPIN mutant (Fig. 5k). The role of TgLIPIN in the production of TAG was further strengthened by the decrease of host-derived C18:1 in TAG (Fig. 5m). In T. gondii parasites, excessive scavenging of C18:1 results in lipotoxicity and parasite death ${ }^{7}$. We show in the absence of TgLIPIN, C18:1 accumulates as FFA and cannot be incorporated into TAG. A significant decrease of C18:1 derived from FASII in the form of FFA is also observed. By sensing this change in nutrients, the parasite attempts to compensate by arresting FASII activity. C18:1 is likely a major FA that is typically scavenged and cannot be stored to avoid lipotoxicity in the absence of TgLIPIN. Taken together, this correlates to previous findings ${ }^{4}$ showing that the parasite is capable of sensing host conditions and metabolically adapt. Similar observations on metabolic plasticity show that there is a reduction in the growth of $P$. falciparum FASII KO mutant in the presence of nutrient-deprived culture conditions ${ }^{4}$. Another study in bacteria shows that the FASII pathway is repressed in a negative feedback loop mediated by acyl-CoA ${ }^{41}$. The mechanisms of metabolic adaptation in Apicomplexa still need to be uncovered. In this study, we were able to reveal the first parasite lipid scavengome, which shows that TgLIPIN prevents parasite 'lipotoxicity' in high nutrient environments through effective channeling of host-scavenged fatty acids while maintaining parasite membrane integrity.

\footnotetext{
Methods

Sequence analysis. TgLIPIN (TGGT1_230690) was identified using EuPathDB web sources ToxoDB (http://toxodb.org/toxo/). A phylogenetic tree of LIPIN proteins in several eukaryotes was created using the online platform Phylogeny.fr. The organisms used for LIPIN protein sequences for generation of the phylogenetic tree include Toxoplasma gondii (TGGT1_230690), Plasmodium falciparum (PF3D7_0303200), P. berghei (PBANKA_040180), Homo sapiens (NX_Q14693), H. sapiens (NX_Q92539), H. sapiens (NX_Q9BQK8), Saccharomyces cerevisiae _PAH1 (PAP1, SMP2, YMR165C, YM8520.14C), Cryptosporidium parvum (cgd3_3210), Cyanidioschyzon merolae (CYME_CMN061C), Neospora caninum (BN1204), Hammondia hammondi (HHA_230690), Chlamydomonas reinhardtii (CHLRE_12g506600v5), Arabidopsis thaliana_AtPAH1 (At3g09560), Arabidopsis thaliana_AtPAH2 (At5g42870), Leishmania major(LMJF_06_0830), Trypanosoma brucei (Tb927.7.5450), Chromera velia (Cvel_24403). The first step involved the curation of these protein sequences. The protein sequences were aligned (ClustalW) and then gaps were removed from the alignment. Finally, the phylogenetic tree was constructed using the maximum likelihood method in the PhyML program (3.1/3.0 aLRT). The default substitution model (WAG) was selected. Graphical representation and edition of the phylogenetic tree were performed with Cladogram.
}

T. gondii strains and cultures. The parasite host cells human foreskin fibroblasts (HFF) were cultured using Dulbecco's Modified Eagle's Medium (DMEM, Gibco) supplemented with $10 \%$ fetal bovine serum (FBS, Gibco), $2 \mathrm{mM}$ glutamine (Gibco), and $25 \mu \mathrm{g} / \mathrm{mL}$ gentamicin (Gibco) at $37^{\circ} \mathrm{C}$ and $5 \% \mathrm{CO}_{2}$.
T. gondii tachyzoite parental strains $\mathrm{RH}-\Delta \mathrm{Ku} 80 \mathrm{TATi}, \mathrm{RH}-\Delta \mathrm{Ku} 80$ as well as mutant strains $T g L I P I N-i k D, T g L I P I N-3 X H A$ were propagated by serial passage within their host HFF using DMEM supplemented with $1 \%$ fetal bovine serum (FBS, Gibco), $2 \mathrm{mM}$ glutamine (Gibco), and $25 \mu \mathrm{g} / \mathrm{mL}$ gentamicin (Gibco) at $37^{\circ} \mathrm{C}$ and $5 \% \mathrm{CO}_{2}$.

Generation of HA-tagged and inducible knockdown line for TgLIPIN. A Cterminally tagged HA line was generated expressing from the gene's endogenous locus using the classical pLIC strategy using homologous recombination in a RH $\Delta \mathrm{Ku} 80$ strain. For the same, a 1677-bp homology region of TgLIPIN located toward to C-terminus excluding the stop codon, was amplified from the parasite genomic DNA using the primers forward $5^{\prime}$-TACTTCCAATCCAATTTAATGC ACGGCAGATTTCTCTTACTGG- $3^{\prime}$ and reverse $5^{\prime}$-TCCTCCACTTCCAATTT TAGCCAAATTACTGCATTTGCGTTCAC-3'. The homology region was assembled into PacI digested pLIC-HA-DHFR plasmid using ligation-independent cloning protocol ${ }^{17}$. The assembled plasmid with linearized with single-enzyme site specific to the parasite DNA sequence within the plasmid-NsiI just before transfection. Parasites were selected with the drug pyrimethamine and cloned by limiting dilution.

For generation of inducible knockdown plasmid pPR2-DHFR ${ }^{42}$, two separate homology flanks were chosen. The $5^{\prime}$ flank was amplified 1637 bp upstream of the TgLIPIN start codon using the primers F1-3' and R1. The PCR product was ligated to PacI and NdeI digested vector pPR2 using NEB assembly reaction. Next, the $3^{\prime}$ flank was amplified as a 1739-bp fragment beginning at the start codon of TgLIPIN with the primers F2 and reverse R2. The $3^{\prime}$ homology flank was annealed to XmaI and NotI digested pPR-HA3-DHFR vector that already contained the TgLIPIN $5^{\prime}$ flank. The final cloned vector positions the start codon of TgLIPIN downstream of the ATc-regulatable t7s4 promoter and a 3xHA tag. The resulting vector with Not and transfected this into TATi $\Delta$ ku 80 parasites. Parasites were selected with the drug pyrimethamine and cloned by limiting dilution.

Screening of parasite clones where the t7s4 promoter had successfully replaced the native TgLIPIN promoter, was done using the screen primers 1-6 in the combinations described in Supplementary Fig. S2. All PCRs were performed with TaKara primestar max polymerase. The knockdown of TgLIPIN was induced with $0.5 \mu \mathrm{g} \mathrm{ml}^{-1}$ of anhydrotetracycline (ATc). Primer list can be found in Supplementary Table 1 .

Immunofluorescence assay. Primary antibodies anti-HA (Rat, Roche 1:500), antiIMC1 (mouse, 1:1000), anti-SAG1 (mouse, 1:1000), anti-MIC2 (rabbit, 1:1000), anti-CPN60 (rabbit, 1:1000), and anti-TOM40 (rabbit, 1:1000) were used at dilutions their respective dilutions. Secondary AlexaFluor 488- and 546-conjugated anti-rat, anti-mouse, and anti-rabbit antibodies (Life Technologies) were used at $1 / 2500$. For the immunofluorescence assay (IFA), parasites were grown on confluent HFF on coverslips and fixed in PBS containing 2.5\% paraformaldehyde (PFA) for $15 \mathrm{~min}$ at room temperature (RT). Samples were permeabilized with $0.25 \%$ Triton X-100 in PBS for $10 \mathrm{~min}$ at RT prior to blocking in PBS containing $3 \%$ BSA and subsequent incubation with primary antibodies then secondary antibodies diluted in the blocking solution. Labeled parasites were stained with Hoechst 33342 (1:10000, Life Technologies) for $20 \mathrm{~min}$ and then washed three times in PBS before final mounting of the coverslips on a glass slide using fluorogel. The IFA slides were visualized using fluorescence microscope (Axio Imager 2_apotome; ZEISS, $\times 60-100$ magnification).

Western blot analysis. Parasites were harvested for western blot analyses after complete egress from their host. To remove any host cell debris, the parasites were passed through a $3-\mu \mathrm{m}$ filter, then counted by haemocytometer and solubilized in SDS buffer at equivalent cell densities. Equal amount of protein was separated on a 4-12\% gradient SDS-polyacrylamide (Life Technologies) and transferred to a nitrocellulose membrane using the XCellII Blot Module (Invitrogen). Primary antibodies anti-HA (rat, Roche) and anti-TOM $40^{43}$ (rabbit) were used at a dilution of 1:500 and 1:1000, respectively. Secondary goat anti-mouse and anti-rabbit horseradish peroxidase (HRP) conjugated antibodies (Thermo Scientific) were used at 1:2000. Protein signal was detected by chemiluminescence after membrane staining with luminata crescendo western HRP detection kit (Millipore). The signal strength of protein was quantified using a BioRad chemidoc imager (BioRad).

Phenotypic analysis. Plaque assay: The extracellular parasites were harvested after filtration and counted by haemocytometer. Then $\sim 500$ parasites were inoculated to a confluent HFF flask $\left(25 \mathrm{~cm}^{2}\right)$. TgLIPIN-ikD was grown for plaque assay in the presence or absence of Anhydrotetracycline (ATc) $\left(0.5 \mu \mathrm{g} \mathrm{ml}^{-1}\right)$ for $7-10$ days. Plaque sizes were visualized by crystal violet staining (30-60 $\mathrm{min})$ after aspiration of culture media, and cells fixation with $100 \%$ ethanol $(5 \mathrm{~min})$ followed by phosphate-buffered saline (PBS) wash. The plaque area was calculated $(n=3)$ using Image J which calculated an arbitrary unit value assessing the viability of the parasite with and without the protein.

Replication assay: The parasites were grown for two days with or without ATc $\left(0.5 \mu \mathrm{g} \mathrm{ml}^{-1}\right)$, harvested and filtered. Equal number of parasites were allowed to invade confluent HFF grown on coverslips. Following $2 \mathrm{~h}$ of invasion, the coverslips were washed thrice with ED1 (1\% FBS containing DMEM), to remove 
extracellular parasites and promote synchronized replication. ATc $\left(0.5 \mu \mathrm{g} \mathrm{ml}^{-1}\right)$ was added at the start of the experiment, allowing the treatment for $24 \mathrm{~h}$, alongside control parasites without ATc. These coverslips were then fixed and processed for IFA using anti-HA, anti-SAG1 antibodies wherein the parasite number per parasitophorous vacuole was analyzed.

Electron microscopy. The TgLIPIN-ikD parasites were grown for 12,24 and $48 \mathrm{~h}$ in the presence and absence of ATc, in labteks (Nunk, Thermofisher). The parental $\Delta \mathrm{Ku} 80$ strain treated with ATc was used as the wild-type control. The labteks containing parasite-infected HFF were fixed in $0.1 \mathrm{M}$ cacodylate buffer with $2.5 \%$ glutaraldehyde for $2 \mathrm{~h}$ and kept at $4{ }^{\circ} \mathrm{C}$ until further processing. During processing, the sample were fixed again for $1 \mathrm{~h}$ with $1 \%$ osmium tetroxide in cacodylate buffer followed by overnight treatment in $2 \%$ uranyl acetate in distilled water. After dehydration in graded series of acetonitrile, samples were progressively impregnated in Epon812, the wells were then filled with fresh resin and allowed to polymerize $48 \mathrm{~h}$ at $60^{\circ} \mathrm{C}$. Ultrathin 70 -nm sections were obtained with a Leica UC7 ultramicrotome and collected on copper grids. Grids were post-stained with uranyl acetate and lead citrate before their observation on a Jeol1200 EXII Transmission Electron Microscope on the Electron microscopy facility of the University of Montpellier (MEA). Chemicals and consumables were from Electron Microscopy Sciences.

Nile red staining of lipid droplets. The TgLIPIN-ikD parasites were allowed to infect and grow in confluent monolayer HFF grown on coverslips in $1 \%$ and $10 \%$ FBS, in the $+/-$ ATc conditions for $24 \mathrm{~h}$ and $48 \mathrm{~h}$. Similar to IFA, these coverslips were fixed using $2.5 \%$ PFA, permeabilized with $0.25 \%$ Triton X-100 and then stained with primary rat anti-HA antibody followed by detection with secondary AlexaFluor 488-conjugated goat anti-rat antibody. Thereafter, the sample coverslips were incubated for $1 \mathrm{~h}$ with Nile red $(1: 2000)$ in $1 \times$ PBS before proceeding to DNA staining with Hoechst. The coverslips were mounted onto a glass slide in fluorogel prior to imaging using a fluorescence microscope (Axio Imager 2_apotome; ZEISS). For visualizing Nile red-stained droplets yellow-gold fluorescence (excitation, $450-500 \mathrm{~nm}$; emission, greater than $528 \mathrm{~nm}^{30}$ ) was used on the axioimager. Quantification in $+/-$ ATc condition was done by counting the number of lipid droplets per parasite vacuole.

Heterologous complementation. The codon-optimized carboxy terminal sequence of TgLIPIN (548-765 a.a., C-LIP) harboring the catalytic HAD domain (DVDGT), obtained from GenScript, was ligated to NotI/MluI digested pD0170 yeast expression vector (obtained from Carman's lab) to yield pD0170-CLIP. This plasmid was transformed into yeast strain $\Delta d p p 1 \Delta l p p 1 \Delta p a h 1$ (pah1_::URA3 $d p p 1 \_:: T R P 1 / K a n r$ lpp1_::HIS3/Kanr, triple mutant) or $\Delta d p p 1 \Delta l p p 1 \Delta p a h 1 \Delta a p p 1$

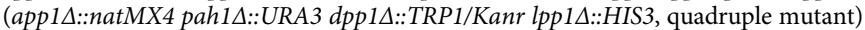
(gifts from Dr. George Carman's lab, Rutgers Center for Lipid Research, New Jersey,), and transformants were selected on solid SD medium (YNB, 2\% glucose, agar) lacking leucine ${ }^{19}$.

\section{Lipidomic analysis}

Saccharomyces cerevisiae. The triple mutant strain was transformed with vector pD0170-CLIP or pDO1050 (lacking the HAD domain) and the cells were grown in YNB glucose medium at $30^{\circ} \mathrm{C}$ until $\mathrm{OD}_{600}$ reached 1-3. Cultures were centrifuged for 5 min at $1000 \times g$, washed with PBS thrice, and normalized by cell weight.

Total lipid analysis: Total lipids were extracted in chloroform/methanol/water $(1: 3: 1, \mathrm{v} / \mathrm{v} / \mathrm{v})$ containing FFA (free fatty acids C13:0, $10 \mathrm{nmol})$ and PC (21:0/21:0, $10 \mathrm{nmol}$ ) as internal standards for extraction. Next, the polar and apolar metabolites were separated by phase partitioning by adding chloroform and water to give the ratio of chloroform/methanol/water as 2:1:0.8 (v/v/v). For lipid analysis, $50 \mu \mathrm{l}$ of the extract was directly dried and dissolved in 2:1 choloform:methanol and trimethylsulfonium hydroxide (TMSH, Machenery Nagel) for total fatty acid content. Resultant FAMEs were then analyzed by GC-MS as previously described ${ }^{44}$. All FAMEs were identified by comparison of retention time and mass spectra from GC-MS with authentic chemical standards. The concentration of FAMEs was quantified after initial normalization to different internal standards.

Phospholipid, DAG/TAG analyses: The extracted total lipid extracted (as above) was separated with $5 \mathrm{nmol}$ DAG/PA(C16:0/C18:1) (Avanti Polar lipids) by onedimensional silica gel high-performance thin-layer chromatography (HPTLC, Merck). The 1st and 2nd solvent system used were chloroform/methanol/water/ acetic acid, 25:15:2:4 (v/v/v/v) and hexane/MTBE/acetic acid, 35:15:0.5 (v/v/v), respectively. For DAG, TAG, FFA, and CE analysis, total lipid fraction was separated by 1D-HPTLC using hexane/diethyl ether/formic acid, 80:20:2 (v/v/v) as solvent system. The spots correlating to TAG, DAG, PA, and PC on the HPTLC plate were scraped off, and lipids were methanolized with $200 \mu \mathrm{l} 0.5 \mathrm{M}$ methanolic $\mathrm{HCl}$ in the presence of $1 \mathrm{nmol}$ pentadecanoic acid (C15:0) as internal standard at $85^{\circ} \mathrm{C}$ for $4 \mathrm{~h}$. The resulting FAMEs were extracted with hexane and analyzed by GC-MS (Agilent).

Toxoplasma gondii. The parasites were grown for $24 \mathrm{~h}$ ad $48 \mathrm{~h}$ in $+/-$ ATc conditions within a confluent monolayer of HFF in flasks $\left(175 \mathrm{~cm}^{2}\right)$. At each time point, parasites were harvested as intracellular tachyzoites $\left(1 \times 10^{7}\right.$ cell equivalents per replicate) after syringe filtration with $3-\mu \mathrm{m}$ pore size membrane. These parasites were metabolically quenched by rapid chilling in a dry ice-ethanol slurry bath and then centrifuged down at $4{ }^{\circ} \mathrm{C}$. The parasite pellet was washed with ice-cold PBS thrice, before transferring the final pellet to a microcentrifuge tube. Then total lipids were extracted in chloroform/methanol/water $(1: 3: 1, \mathrm{v} / \mathrm{v} / \mathrm{v})$ containing PC (C13:0/C13:0), $10 \mathrm{nmol}$ and $\mathrm{C} 21: 0(10 \mathrm{nmol})$ as internal standards for extraction. Polar and apolar metabolites were separated by phase partitioning by adding chloroform and water to give the ratio of chloroform/methanol/water as 2:1:0.8 $(\mathrm{v} / \mathrm{v} / \mathrm{v})$. For lipid analysis, the organic phase was dried under $\mathrm{N}_{2}$ gas and dissolved in 1-butanol to obtain $1 \mu \mathrm{l} \mathrm{butanol} / 10^{7}$ parasites.

Total lipid analysis: The extracted total lipid sample was then added with 1 nmol pentadecanoic acid (C15:0) as an internal standard as stated before using TMSH for total fatty acid content. Resultant FAMEs were analyzed by GC-MS as previously described ${ }^{44}$. All FAMEs were identified by comparison of retention time and mass spectra from GC-MS with authentic chemical standards. The concentration of FAMEs was quantified after initial normalization to different internal standards and finally to parasite number.

Free fatty acid and cholesterol analysis: Total lipid samples were dried and derivatized with BSTFA + TMCS, 99:1 (Sigma) to generate trimethylsilyl (TMS-) fatty acids and TMS-cholesterol. These TMS derivatives were analyzed by GCMS as described above.

Phospholipid and neutral lipid analysis: For phospholipid analysis, the extracted total lipid extracted (as above) was separated with $1 \mathrm{nmol} \mathrm{PA}(\mathrm{C} 17: 0 / \mathrm{C} 17: 0)$ (Avanti Polar lipids) by two-dimensional silica gel high-performance thin-layer chromatography (HPTLC, Merck). The solvent system used for the 1st and 2nd dimension was chloroform/methanol/28\% ammonium hydroxide,12:7:1.6 (v/v) and chloroform/acetone/methanol/acetic acid/water, 10:4:2:2.6:1 (v/v/v/v/v), respectively. For DAG, TAG, Free fatty acids (FFA) and cholesteryl ester (CE) analysis, total lipid fraction was separated by 1D-HPTLC using hexane/diethyl ether/formic acid, 80:20:2 (v/v/v) as solvent system. Then each lipid spot on the HPTLC plate was scraped off, and lipids were methanolized with $200 \mu \mathrm{l} 0.5 \mathrm{M}$ methanolic $\mathrm{HCl}$ in the presence of $1 \mathrm{nmol}$ pentadecanoic acid (C15:0) as interna standard at $85^{\circ} \mathrm{C}$ for $3 \mathrm{~h}$. The resulting FAMEs were extracted with hexane and analyzed by GC-MS (Agilent).

\section{Stable isotope metabolic labeling experiment}

Tracking FASII origin fatty acids (monitoring de novo FA synthesis by the parasite apicoplast FASII). The TgLIPIN parasites were infected to a confluent monolayer of HFF in glucose-free-DMEM ( $1 \%$ FBS) supplemented with $\mathrm{U}^{13} \mathrm{C}$-glucose or $\mathrm{U}$ -

${ }^{12} \mathrm{C}$-glucose at a final concentration of $800 \mu \mathrm{M}$, with or without ATc $\left(0.5 \mu \mathrm{g} \mathrm{ml}^{-1}\right)$. The parasites were harvested up to $48 \mathrm{~h}$ post depletion of TgLIPIN and metabolically quenched in a dry ice and ethanol slurry in a tube until the sample reached $4^{\circ} \mathrm{C}$. Lipids were extracted, derivatized using TMSH (Macherey-Nagel) and analyzed by GC-MS as described above. ${ }^{13} \mathrm{C}$ incorporation to each fatty acid was calculated as the percent of the metabolite pool containing one or more ${ }^{13} \mathrm{C}$ atoms after correction for natural abundance and the amount of ${ }^{13} \mathrm{C}$-carbon source in the culture medium. The degree of the incorporation of ${ }^{13} \mathrm{C}$ into fatty acids (\% carbon incorporation) was determined by the mass isotopomer distribution (MID) of each FAMEs. MID was obtained from the shift in isotopic mass dependent on the amount of ${ }^{12} \mathrm{C}$ carbons compared to the integration of ${ }^{13} \mathrm{C}$ carbon atoms. The total abundance of ${ }^{13} \mathrm{C}$-labeled fatty acids was obtained by calculating the concentration of all isotopomers of ${ }^{13} \mathrm{C}$-labeled FAMEs and finally normalizing to authentic internal standards and parasite number.

Tracking host-derived fatty acids (monitoring parasite scavenging capacities). The HFF cells were grown $\left(1 \times 10^{8}\right.$ cell equivalents per replicate) to confluency in the presence of stable isotope $\mathrm{U}^{13} \mathrm{C}$-glucose at a final concentration of $800 \mu \mathrm{M}$ added to a glucose-free DMEM. These ${ }^{13} \mathrm{C}$-pre-labeled HFF were then infected with TgLIPIN-ikD parasites in the presence of normal-glucose containing DMEM under $+/-\operatorname{ATc}(0.5 \mu \mathrm{g} / \mathrm{ml})$. The host HFF and parasites were metabolically quenched separately, and their lipid content was quantified by GC-MS as described above. As described previously, the degree of the incorporation of ${ }^{13} \mathrm{C}$ into fatty acids (\%carbon incorporation) is determined by the mass isotopomer distribution (MID) of each FAMEs. The total abundance of ${ }^{33} \mathrm{C}$-labeled fatty acids was analyzed initially for HFF to check labeling of the metabolites (described previously). Later, the same was calculated for parasites to confirm direct uptake of ${ }^{13} \mathrm{C}$-labeled fatty acids from the host.

Tracking uptake of deuterated fatty acid from medium (monitoring extracellular host environment). Deuterated palmitic acid $\left({ }^{31} \mathrm{~d}-\mathrm{C} 16: 0\right)$ was dissolved in $10 \mathrm{mM}$ in fatty acid-free bovine serum albumin/PBS solution by sonication in a water bath for $30 \mathrm{~min}$ followed by incubation at $55^{\circ} \mathrm{C}$ for $30 \mathrm{~min}$. Freshly egressed TgLIPIN parasites were allowed to invade a confluent monolayer of HFF for at least $2 \mathrm{~h}$ under conditions of $+/-$ ATc. Following the invasion, the uninvaded parasites were washed off with DMEM and further allowed to grow in the normal culture medium-DMEM (1\% FBS) containing ${ }^{31} \mathrm{~d}-\mathrm{C} 16: 0$ at a final concentration of $0.1 \mathrm{mM}$ in $+/-$ ATc until 24 and $48 \mathrm{~h}$ of growth. The parasites were harvested by metabolic quenching as described previously. Lipids were extracted, derivatized using TMSH as well as TMS, and further analyzed by GC-MS. All raw data were quantitatively and qualitatively analyzed using Agilent MassHunter Software: MS B.07.00 
Statistics and reproducibility. The graphical data for this study was generated using GraphPad Prism software. Three biological replicates were used per experiment ( $n=3$, unless stated otherwise). The error bars are representative of the standard error of mean (SEM) for each study. Statistical significance was determined for each experiment by unpaired $t$ tests using GraphPad Prism. The range of statistical significance was signified as per the $P$ value, where $0.01-0.05=*$, $0.01-0.001=* *,<0.001=* * *$, and $<0.0001=* * * *$

Reporting summary. Further information on research design is available in the Nature Research Reporting Summary linked to this article.

\section{Data availability}

Authors can confirm that all relevant data are included in the paper and/or its Supplementary/Source data files. Protein and gene sequences were acquired from EuPathDB web sources ToxoDB (http://toxodb.org/toxo/). Source data are provided with this paper.

\section{Code availability}

No codes were used to analyze the data in this study.

Received: 19 February 2020; Accepted: 24 March 2021;

Published online: 17 May 2021

\section{References}

1. Ramakrishnan, S., Serricchio, M., Striepen, B. \& Bütikofer, P. Lipid synthesis in protozoan parasites: a comparison between kinetoplastids and apicomplexans. https://doi.org/10.1016/j.plipres.2013.06.003 (2013).

2. Ramakrishnan, S. et al. The intracellular parasite Toxoplasma gondii depends on the synthesis of long chain and very long-chain unsaturated fatty acids not supplied by the host cell HHS public access. Mol. Microbiol. 97, 64-76 (2015).

3. Coppens, I. Targeting lipid biosynthesis and salvage in apicomplexan parasites for improved chemotherapies. Nat. Rev. Microbiol. 11, 823-835 (2013).

4. Amiar, S. et al. Division and adaptation to host environment of apicomplexan parasites depend on apicoplast lipid metabolic plasticity and host organelle remodeling. Cell Rep. 30, 3778-3792 (2020).

5. Di Genova, B. M., Wilson, S. K., Dubey, J. P. \& Knoll, L. J. Intestinal delta-6desaturase activity determines host range for Toxoplasma sexual reproduction. PLoS Biol. 17, 1-19 (2019).

6. Mazumdar, J. \& Striepen, B. Make it or take it: fatty acid metabolism of apicomplexan parasites. Eukaryot. Cell 6, 1727-1735 (2007).

7. Nolan, S. J., Romano, J. D., Kline, J. T. \& Coppens, I. Novel approaches to kill Toxoplasma gondii by exploiting the uncontrolled uptake of unsaturated fatty acids and vulnerability to lipid storage inhibition of the parasite. Antimicrob. Agents Chemother. 62, e00347-18 (2018).

8. Csaki, L. S. \& Reue, K. Lipins: multifunctional lipid metabolism proteins. Annu. Rev. Nutr. 30, 257-272 (2010).

9. Bullen, H. E. et al. Phosphatidic acid-mediated signaling regulates microneme secretion in toxoplasma. Cell Host Microbe 19, 349-360 (2016).

10. Jacot, D. et al. An apicomplexan actin-binding protein serves as a connector and lipid sensor to coordinate motility and invasion. Cell Host Microbe 20, 731-743 (2016).

11. Bisio, H., Lunghi, M., Brochet, M. \& Soldati-Favre, D. Phosphatidic acid governs natural egress in Toxoplasma gondii via a guanylate cyclase receptor platform. Nat. Microbiol. https://doi.org/10.1038/s41564-018-0339-8 (2019).

12. Jimah, J. R. et al. Malaria parasite CelTOS targets the inner leaflet of cell membranes for pore-dependent disruption. eLife https://doi.org/10.7554/ eLife.20621 (2016).

13. Amiar, S. et al. Apicoplast-localized lysophosphatidic acid precursor assembly is required for bulk phospholipid synthesis in Toxoplasma gondii and relies on an algal/plant-like glycerol 3-phosphate acyltransferase. PLoS Pathog. 12, 1-30 (2016).

14. Carman, G. M. \& Han, G. S. Fat-regulating phosphatidic acid phosphatase: a review of its roles and regulation in lipid homeostasis. J. Lipid Res. 60, 2-6 (2019).

15. Gupta, N., Zahn, M. M., Coppens, I., Joiner, K. A. \& Voelker, D. R. Selective disruption of phosphatidylcholine metabolism of the intracellular parasite Toxoplasma gondii arrests its growth. J. Biol. Chem. 280, 16345-16353 (2005).

16. Déchamps, S., Shastri, S., Wengelnik, K. \& Vial, H. J. Glycerophospholipid acquisition in Plasmodium-A puzzling assembly of biosynthetic pathways. Int. J. Parasitol. 40, 1347-1365 (2010).

17. Carman, G. M. \& Han, G. S. Phosphatidic acid phosphatase, a key enzyme in the regulation of lipid synthesis. J. Biol. Chem. https://doi.org/10.1074/jbc. R800059200 (2009).
18. Nakamura, Y. et al. Arabidopsis lipins mediate eukaryotic pathway of lipid metabolism and cope critically with phosphate starvation. Proc. Natl Acad. Sci. USA 106, 20978-20983 (2009).

19. Chae, M., Han, G. S. \& Carman, G. M. The Saccharomyces cerevisiae actin patch protein applp is a phosphatidate phosphatase enzyme. J. Biol. Chem. 287, 40186-40196 (2012).

20. Huynh, M. H. \& Carruthers, V. B. Tagging of endogenous genes in a Toxoplasma gondii strain lacking Ku80. Eukaryot. Cell 8, 530-539 (2009).

21. Zhang, P. \& Reue, K. Lipin proteins and glycerolipid metabolism: roles at the ER membrane and beyond. Biochimica et Biophysica Acta - Biomembranes https://doi.org/10.1016/j.bbamem.2017.04.007 (2017).

22. Agrawal, S., Dooren, G. G. Van, Beatty, W. L. \& Striepen, B. Genetic evidence that an endosymbiont-derived endoplasmic reticulum-associated protein degradation (ERAD) system functions in import of apicoplast proteins. J. Biol. Chem. 284, 33683-33691 (2009).

23. Sheiner, L. et al. A systematic screen to discover and analyze apicoplast proteins identifies a conserved and essential protein import factor. PLoS Pathog. 7, e1002392 (2011).

24. Soldati, D., Meissner, M. \& Schluter, D. Role of Toxoplasma gondii myosin A in powering parasite gliding and host cell invasion. Science 298, 837-840 (2002).

25. Santos-Rosa, H., Leung, J., Grimsey, N., Peak-Chew, S. \& Siniossoglou, S. The yeast lipin Smp2 couples phospholipid biosynthesis to nuclear membrane growth. EMBO J. https://doi.org/10.1038/sj.emboj.7600672 (2005).

26. Siniossoglou, S. Lipins, lipids and nuclear envelope structure. Traffic https:// doi.org/10.1111/j.1600-0854.2009.00923.x (2009).

27. Kong, P. et al. Two phylogenetically and compartmentally distinct CDPdiacylglycerol synthases cooperate for lipid biogenesis in Toxoplasma gondii. J. Biol. Chem. https://doi.org/10.1074/jbc.M116.765487 (2017).

28. Cases, S. et al. Identification of a gene encoding an acyl CoA:diacylglycerol acyltransferase, a key enzyme in triacylglycerol synthesis. Proc. Natl Acad. Sci. USA https://doi.org/10.1073/pnas.95.22.13018 (1998).

29. Nolan, S. J., Romano, J. D. \& Coppens, I. Host lipid droplets: an important source of lipids salvaged by the intracellular parasite Toxoplasma gondii. PLoS Pathogens 13, e1006362 (2017).

30. Greenspan, P., Mayer, E. P. \& Fowler, S. D. Nile red: a selective fluorescent stain for intracellular lipid droplets. J. Cell Biol. 100, 965-973 (1985).

31. Hu, X., Binns, D. \& Reese, M. L. The coccidian parasites Toxoplasma and Neospora dysregulate mammalian lipid droplet biogenesis. J. Biol. Chem. 292, 11009-11020 (2017).

32. Mashima, T., Seimiya, H. \& Tsuruo, T. De novo fatty-acid synthesis and related pathways as molecular targets for cancer therapy. Br. J. Cancer https:// doi.org/10.1038/sj.bjc.6605007 (2009).

33. Reue, K. \& Wang, H. Mammalian lipin phosphatidic acid phosphatases in lipid synthesis and beyond: Metabolic and inflammatory disorders. J. Lipid Res. https://doi.org/10.1194/jlr.S091769 (2019).

34. Karanasios, E., Han, G. S., Xu, Z., Carman, G. M. \& Siniossoglou, S. A phosphorylation-regulated amphipathic helix controls the membrane translocation and function of the yeast phosphatidate phosphatase. Proc. Natl Acad. Sci. USA https://doi.org/10.1073/pnas.1007974107 (2010).

35. Kim, Y. et al. A conserved phosphatase cascade that regulates nuclear membrane biogenesis. Proc. Natl. Acad. Sci. USA https://doi.org/10.1073/ pnas.0702099104 (2007)

36. Pascual, F. \& Carman, G. M. Phosphatidate phosphatase, a key regulator of lipid homeostasis. Biochim. Biophys. Acta 1831, 514-522 (2013).

37. Han, G. S., O'Hara, L., Carman, G. M. \& Siniossoglou, S. An unconventional diacylglycerol kinase that regulates phospholipid synthesis and nuclear membrane growth. J. Biol. Chem. 283, 20433-20442 (2008).

38. Barbosa, A. D. et al. Lipid partitioning at the nuclear envelope controls membrane biogenesis. Mol. Biol. Cell https://doi.org/10.1091/mbc.E15-030173 (2015)

39. Listenberger, L. L. et al. Triglyceride accumulation protects against fatty acidinduced lipotoxicity. Proc. Natl Acad. Sci. USA https://doi.org/10.1073/ pnas.0630588100 (2003).

40. Pernas, L., Bean, C., Boothroyd, J. C. \& Scorrano, L. Mitochondria restrict growth of the intracellular parasite Toxoplasma gondii by limiting its uptake of fatty acids. Cell Metab. 27, 886-897.e4 (2018).

41. Fujita, Y., Matsuoka, H. \& Hirooka, K. Regulation of fatty acid metabolism in bacteria. Mol. Microbiol. https://doi.org/10.1111/j.1365-2958.2007.05947.x (2007).

42. Katris, N. J. et al. The apical complex provides a regulated gateway for secretion of invasion factors in toxoplasma. PLoS Pathog. 10, e1004074 (2014).

43. Van Dooren, G. G., Yeoh, L. M., Striepen, B. \& McFadden, G. I. The import of proteins into the mitochondrion of Toxoplasma gondii. J. Biol. Chem. 291, 19335-19350 (2016).

44. Dubois, D. et al. Toxoplasma gondii acetyl-CoA synthetase is involved in fatty acid elongation (of long fatty acid chains) during tachyzoite life stages. J. Lipid Res. 59, 994-1004 (2018). 


\section{Acknowledgements}

We would like to thank Prof George M. Carman for providing us the yeast mutant with corresponding plasmids. This work and C.Y.B., Y.Y.B., S.D., S.S., C.S.A., and N.J.K. are supported by Agence Nationale de la Recherche, France (Grant ANR-12-PDOC-0028Project Apicolipid), the Atip-Avenir and Finovi programs (CNRS-INSERM-FinoviAtipAvenir Apicoplipid projects), CEFIPRA (Indo-French Centre for the Promotion of Advanced Research), IDEX-Université Grenoble Alpes, Region Auvergne Rhone-Alpes (IRICE Grant, Project GEMELI) and the Laboratoire d'Excellence ParaFrap, France (Grant number ANR-11-LABX-0024).

\section{Author contributions}

C.Y.B., Y.Y.B., and S.D. contributed to the design of the work. C.Y.B., Y.Y.B., L.B., S.S., F.B., M.F.C., and S.Da. contributed to the interpretation of data. S.Da., S.S., S.Du., F.B., C.S.A., N.J.K., L.B., Y.Y.B., and C.Y.B. contributed the acquisition and analysis of data. M.F.C. and C.Y.B. financed the project.

\section{Competing interests}

The authors declare no competing interests.

\section{Additional information}

Supplementary information The online version contains supplementary material available at https://doi.org/10.1038/s41467-021-22956-w.

Correspondence and requests for materials should be addressed to Y.Y-B. or C.Y.B.
Peer review information Nature Communications thanks Laura Knoll, Rachel Zufferey, and the other, anonymous reviewer(s) for their contribution to the peer review of this work.

Reprints and permission information is available at http://www.nature.com/reprints

Publisher's note Springer Nature remains neutral with regard to jurisdictional claims in published maps and institutional affiliations.

(c) (i) Open Access This article is licensed under a Creative Commons Attribution 4.0 International License, which permits use, sharing, adaptation, distribution and reproduction in any medium or format, as long as you give appropriate credit to the original author(s) and the source, provide a link to the Creative Commons license, and indicate if changes were made. The images or other third party material in this article are included in the article's Creative Commons license, unless indicated otherwise in a credit line to the material. If material is not included in the article's Creative Commons license and your intended use is not permitted by statutory regulation or exceeds the permitted use, you will need to obtain permission directly from the copyright holder. To view a copy of this license, visit http://creativecommons.org/ licenses/by/4.0/.

(C) The Author(s) 2021 